\title{
SENSOR NETWORK DESIGN FOR THE ESTIMATION OF SPATIALLY DISTRIBUTED PROCESSES
}

\author{
DARIUSZ UCIŃSKI, MACIEJ PATAN
}

Institute of Control and Computation Engineering

University of Zielona Góra, ul. Podgórna 50, 65-246 Zielona Góra, Poland

e-mail:\{d.ucinski,m.patan\}@issi.uz.zgora.pl

\begin{abstract}
In a typical moving contaminating source identification problem, after some type of biological or chemical contamination has occurred, there is a developing cloud of dangerous or toxic material. In order to detect and localize the contamination source, a sensor network can be used. Up to now, however, approaches aiming at guaranteeing a dense region coverage or satisfactory network connectivity have dominated this line of research and abstracted away from the mathematical description of the physical processes underlying the observed phenomena. The present work aims at bridging this gap and meeting the needs created in the context of the source identification problem. We assume that the paths of the moving sources are unknown, but they are sufficiently smooth to be approximated by combinations of given basis functions. This parametrization makes it possible to reduce the source detection and estimation problem to that of parameter identification. In order to estimate the source and medium parameters, the maximum-likelihood estimator is used. Based on a scalar measure of performance defined on the Fisher information matrix related to the unknown parameters, which is commonly used in optimum experimental design theory, the problem is formulated as an optimal control one. From a practical point of view, it is desirable to have the computations dynamic data driven, i.e., the current measurements from the mobile sensors must serve as a basis for the update of parameter estimates and these, in turn, can be used to correct the sensor movements. In the proposed research, an attempt will also be made at applying a nonlinear model-predictive-control-like approach to attack this issue.
\end{abstract}

Keywords: optimal experiment design, parameter estimation, sensor network, source identification.

\section{Introduction}

1.1. Motivations behind the source identification problem. The contaminating source identification problem has been increasingly receiving significant research interest due to its applications in the fields of security, environmental and industrial monitoring, pollution control, etc. Examples include the detection of a potential biochemical attack in an urban environment, e.g., from a crop-duster spreading toxins in aerosol, sensing explosives mounted on a vehicle, detecting a leakage of dangerous biochemical materials from a tank carried by a vehicle, or detecting a life-threatening contaminant source dropped intentionally or unintentionally into a water reservoir. In a typical scenario, after some type of biological or chemical contamination has occurred, there is a developing plume of dangerous or toxic material. Clearly, the evolution of the cloud will be affected by the weather conditions and the surrounding geography. It goes without saying that a crucial factor in responding to a chemical or biological attack is speed. Emergency services wish to quickly know what the toxin is, where its sources are located and what their strengths are. This knowledge would allow them to take the appropriate measures to counteract or at least to reduce the impact of the release.

Although laborious research is conducted towards technologies making it possible to remotely detect the presence of a toxin, it is rather hard to envisage systems continuously monitoring vast spatial regions over long time horizons. But even with extremely limited measurement resources in space and time, the identification of contamination sources is not doomed to failure, provided that one has ample knowledge of the diffusion field and wind velocity. This is because there exist mathematical models that govern the development of the plume. They are based on partial differential equations which include diffusion and transport phenomena effects, as well as forcing functions such as the prevailing weather conditions, or boundary conditions related to the possibly complicated sur- 
rounding geography. Now, assume that a number of sensors is deployed in the spatial area in question and they can measure the concentration of the contaminant. Then the goal is to use these observations to detect and localize the contamination source, determine the space-time concentration distribution of the chemical dispersion, and predict its cloud envelope evolution. As far as the form of the mathematical model for the plume evolution is known, a model-based approach can be used, wherein the underlying physical and statistical models models are coupled with signal processing to obtain the best performance.

There are a number of difficulties inherent in the above source identification problem. First, we note that this is an inverse problem in which, given a model, it is necessary to identify the system parameters from available information about the process. As such, we demand certain guarantees that the source position is identifiable taking account of the typical case when sensors permit finite-dimensional measurements. Second, as a rule, this inverse problem is ill-posed and has to be regularized to obtain a reasonable approximation to the solution. This question can hardly be overestimated, since, using observations provided by sensors which are usually coarsely spaced and without resorting to regularization, we cannot hope to recover components of the source function that are more oscillatory than dictated by the spacing of the sensors. These issues are often neglected in the engineering literature, which may lead to erroneous results, $\mathrm{cf}$. the work of Sivergina and Polis (2002).

The situation is even more complicated when there are multiple toxic sources which can move and keep polluting the zone in question. Then, additionally, from the online implementation viewpoint, we would prefer a recursive procedure for estimating time-dependent parameters characterizing the sources so that the estimate tracks the measurement data and so the new measurements can be effectively incorporated. Unfortunately, no universal solutions to the above problems have been proposed so far.

1.2. Sensor management. In modern observation systems, sensors can be located on various platforms and these platforms can be highly dynamic in motion. Recent advances in hardware, sensor and networking technologies enable large-scale deployment of superior data acquisition systems with adjustable resolutions, called sensor networks (Zhao and Guibas, 2004; Hirsch et al., 2008; Jain and Agrawal, 2005; Sastry and Iyengar, 2005; Chong and Kumar, 2003; Sinopoli et al., 2003; Cassandras and Li, 2005). Each sensor node has a sensing capability, as well as limited energy supply, computing power, memory and communication ability. These inexpensive, low-power communication devices can be deployed throughout the physical space, providing dense sensing close to physical phenomena, processing and communicating this infor- mation, and coordinating actions with other nodes. Sensor networks have recently come into prominence because they hold the potential to revolutionize a wide spectrum of both civilian and military applications, including environmental monitoring, scene reconstruction, motion tracking, motion detection, battlefield surveillance, remote sensing, global awareness, etc. The design, implementation and operation for a sensor network requires the confluence of many disciplines, including signal processing, networking and protocols, embedded systems, information management and distributed algorithms.

Endowing nodes in a sensor network with mobility drastically expands the spectrum of the network's capabilities. Moreover, assuming that each mobile node possesses a certain amount of decision making autonomy gives rise to a dynamic system with a considerable amount of flexibility, depending on the extent to which the nodes can cooperate in order to perform a mission. This flexibility, for example, allows us to handle a large number of data source targets with a much smaller number of nodes that can move. What is more, technological advances in communication systems and the growing ease in making small, low power and inexpensive mobile systems now make it feasible to deploy a group of networked vehicles in a number of environments (Ögren et al., 2004; Chong and Kumar, 2003; Sinopoli et al., 2003; Cassandras and Li, 2005; Martínez and Bullo, 2006).

In a simplest case, each networked sensor can be mounted on a ground mobile robot. The mission in context is to determine possibly multiple contamination sources. Each robot is actuated according to spatial and temporal sensed information (contaminant concentration gradient, spatial position, etc.) from more than one mobile sensor. A more complicated scenario consists in exploring a threedimensional space using mobile sensors being unmanned aerial vehicles (UAVs) equipped with concentration detectors. Using all possible data (such as weather information, information about the storage facility and the possible hazardous materials that have been released), the command and control system determines where sensor measurements should be made and directs the mobile sensors to appropriate sampling locations.

Naturally, mobility implies an additional layer of complexity (Cassandras and Li, 2005). For example, if communication connectivity is to be maintained, we must ensure that each node remains within the range of at least some other nodes. We must also take into account that mobility consumes a considerable amount of energy, which amplifies the need for various forms of power control. However, the complexity of the resulting sensor management problem is compensated by a number of benefits. Specifically, sensors are not assigned to fixed spatial positions, but are capable of tracking points which provide at a given time moment the best information about the contamination sources. In order to take advantage of these possibi- 
lities, sensors must be deployed and then guided so as to maximize the information extracted from the mission space while maintaining acceptable levels of communication and energy consumption.

1.3. Previous work. The input reconstruction problem has extensively been investigated since the 1960s due to its importance in military applications and environmental monitoring. Most approaches pass a reference to a general framework of inverse problems for partial differential equations (cf. Isakov, 1998). Among recent works we can enumerate, e.g., the one by Sivergina et al. (2003), where a recursive identification procedure was exposed based on estimation theory for PDEs with bounded disturbances and Tikhonov regularization techniques (specifically, a generalization of a finite-dimensional Lyapunov technique for systems with bounded disturbances is considered). This scheme is closely related to the on-line approach by Maksimov (2000), resting on principles of positional control, which was investigated in various settings and developed for linear parabolic and hyperbolic equations, nonlinear equations with monotone operators, and variational inequalities. Note, however, that those technically sound methods were tested only on academic examples. On the other hand, a number of contributions appeared aimed at source identification in complex settings encountered in practice.

Thus, Akçelik et al. (2003) investigated the reconstruction of an arbitrary source in a time-varying convective-diffusive transport in three space dimensions, given a velocity field and pointwise measurements of concentration. The source identification problem is converted to an infinite-dimensional optimization one for which optimality conditions are derived and solved using a Galerkin space-time finite-element method using a terascale supercomputer. In turn, Boggs et al. (2006) proposed a similar formulation for rapid source inversion for a steadystate environment in which the transport is dominated by advection and the turbulent flow is modeled using a Reynolds-averaged Navier-Stokes approach.

In the work by Gnot et al. (2001), a method was developed to identify one pollution source acting on a static DPS described by an elliptic equation. Based on measurements from several monitoring stations, the authors set forth a statistical inference procedure to estimate the mean and variance of the source intensity, along with the variance of the measurement errors. Separating variance components is usually avoided in the literature on source identification and therefore the value of this original technique can hardly be overestimated.

The number of publications on mobile sources is limited owing to the inevitable increase in problem complexity. Indeed, transition from static to mobile sources may lead to states of a DPS which cannot be achieved when the forcing input is by non-mobile sources only and, in con- sequence, affect the underlying system properties. What is more, this transition yields a loss of linearity in the basic relations, see the work of Butkovskiy and Pustyl'nikov (1987) for more details. In spite of that, the problem was attacked from various angles. Zhao and Nehorai (2006) developed a statistical method to detect and estimate biochemical dispersion by a moving source using modelbased integrated sensor-array processing. After parameterizing the unknown quantities (e.g., the source intensity and trajectory) by a finite number of constant parameters, close attention is paid to the detection and localization of the biochemical source using maximum-likelihood estimators. Additionally, the Cramér-Rao bound is computed to assess the achieved results. What is more, detectors are constructed based on a generalized likelihood ratio test (GLRT), and their performance is determined in terms of the probabilities of detection and a false alarm. The presented approach constitutes a generalization of the method set forth by Nehorai et al. (1995) for the case of stationary sensors and sources, and then extended by Porat and Nehorai (1996) to the case of a moving sensor.

The identification of a moving source for a parabolic equation with constant coefficients was attacked from a different perspective by Kusiak and Weatherwax (2008). The authors demonstrate that a knowledge of the instantaneous concentration distribution of the dispersion on any bounded open set located away from the support of a source is sufficient to estimate a nontrivial subset of the actual convex hull of the support of the source which they call the carrier support.

The estimation of moving point sources in a threedimensional homogeneous solid in transient heat conduction was investigated by Lefèvre and Niliot (2002). The identification procedure is based on a boundary element method formulation using transient fundamental solutions. The results were verified on both simulation and experimental examples.

1.4. Our work. A detailed review of the existing approaches provides evidence that no satisfactory and versatile method to detect and estimate moving sources has been proposed as yet, at least not for applications where (near) real-time solutions are needed (i.e., sensor data have to be assimilated repeatedly into simulations). Above all, this is because the size and complexity of PDE simulations often present significant computational challenges (Biegler et al., 2007). It also goes without saying that the relevant algorithms are expected to produce acceptable approximations to the sought values fast enough to make timely decisions counteracting the aftermaths of chemical or biological attacks. What is more, the prospective methods must be capable of dealing with the inherent illposedness of inverse problems and robust to incomplete, uncertain and noisy data. This is in sharp contrast to the literature, where increasingly complex parameterized mo- 
dels are treated theoretically and computationally in a deterministic framework with little or no attention to uncertainty in either the modeled mechanisms or the data used to validate the models (Vogel, 2002). Finally, to the best of our knowledge, no communications have been reported regarding sensor trajectory generation for the purpose of an "optimal" estimation of moving sources. Inevitably, node mobility involves an additional level of complexity, but this measurement strategy holds much appeal from the perspective of achievable identification accuracy and, what is crucial here, it can be easily implemented using current technology.

The purpose of the research pursued in this work was thus to develop a computationally efficient approach to the detection and estimation of moving contamination sources using a sensor network with multiple cooperative mobile nodes. In contrast to conventional approaches commonly used in mobile sensor networks, the knowledge of the mathematical model of the distributed parameter system in question is incorporated to form a basis for optimization. Consequently, the important information about the model governing the physical phenomenon in question will not be lost and will be to the profit of early detection of potential chemical and biological threats. The main idea applied here consists in formulating the problem in terms of a statistical measurement model.

\subsection{Proposed approach.}

1.5.1. Role of the PDE model in configuring sensor networks with mobile nodes. In a typical sensor network application, sensors are supposed to be deployed so as to monitor a region and collect the most valuable information from the observed system. The quality of sensor deployment can be quantified by the appropriate performance indices, and optimum sensor node configurations can thus be sought. The resulting observation strategies concern optimal planning of trajectories of mobile nodes. Up to now, approaches aiming at guaranteeing a dense region coverage or satisfactory network connectivity have dominated this line of research and abstracted away from the mathematical description of the physical processes underlying the observed phenomena. In this way, much information is lost which could potentially be used to make the operation of the sensor network more efficient and yield substantial gains in the functionality of the whole source localization system.

The observed processes in question are often termed distributed parameter systems (DPSs) as their states depend not only on time, but also on spatial coordinates. Appropriate mathematical modeling of DPSs most often yields partial differential equations (PDEs). It goes without saying that such models involve using rather sophisticated mathematical methods. This explains why so few attempts have been made at exploiting them in the context of sensor networks. But in recompense for this effort, we would be in a position to describe the process more accurately and to implement more effective control strategies. Early lumping, which means the approximation of PDEs by ordinary differential equations of possibly high order, may mask the distributed nature of the system and therefore it is not always satisfactory.

For the last forty years, DPSs have occupied an important place in control and system theories. They are now an established area of research with a long list of journal articles, conference proceedings and numerous textbooks. It is intriguing that for a long time, due to the inherent impossibility of observing the system states over the entire spatial domain, one of the topics of importance for specialists in control theory has been the problem of selecting the number and location of sensors and actuators for the control and state/parameter estimation in such systems. A number of sensor location methods were invented and supported by a sound theory, but they are not directly fit to the emerging technology of sensor networks. The present work aims at bridging this gap and meeting the needs created in the context of the source identification problem.

\subsubsection{Problem formulation in terms of a statistical} measurement model. Suppose that we have a number of unknown moving sources (intruders) continuously releasing a contaminant. In the proposed approach, we start with the physical model including a PDE describing the phenomenon of contaminant advection and diffusion over a spatial domain. In some situations, some information about the source trajectories may be known. For example, the moving source path can be known in advance for a car in streets or a train on rails. For an airplane, the moving trajectory can be known by radar. But in a general case which is considered here, we assume that the path of each moving source is unknown. Basically, it is possible to model it by independent spatial samples at each time snapshot. However, the resulting number of unknown parameters (being spatial positions at consecutive time moments) to estimate would be excessively high. Accordingly, the computational costs would be extremely high and the results would be inaccurate. Therefore, the use of a parametric moving path model was advocated by Zhao and Nehorai (2006). Such a model exploits the fact that the trajectories are smooth, which makes it possible to somewhat reduce the number of parameters to be estimated. In a simplest case, each path could be approximated by a linear combination of a set of given basis functions. These temporal basis functions should be chosen based on prior information on the trajectory. Their number should be chosen as a trade-off between the desired accuracy and computational complexity. Zhao and Nehorai (2006) used the following reasoning: If a sour- 
ce trajectory is smooth, a comparatively small number of basis functions and unknown parameters can be used to construct a sufficiently good approximation to its path. Consequently, they developed a sound approach to source identification, which was thoroughly analyzed and tested on simulation examples. Although the parameterized model sounds appealing, as its introduction reduces the problem to a parameter identification one, the reality is that the number of the relevant parameters to be estimated may still be too high to avoid acute problems involved by the extreme ill-posedness of the nonlinear least-squares approach (cf. Banks, 1992; Chavent, 1991), e.g., the lack of the uniqueness of solutions, instabilities in the minimization process or the presence of many local minima. These, in turn, create a need for appropriate regularization procedures, which results in an increased complexity level.

Consequently, we have decided to alter the basic parameter estimation procedure outlined above and to decompose source identification into a sequence of stages carried out over consecutive time intervals. If these contiguous intervals are short enough, then it is reasonable to assume that over each of them sources move along straight lines at constant speeds and release the contaminant at constant rates. We handle the parameters governing this simplified source behavior by estimating them off-line based on the measurement data collected over the current time stage. To a certain extent, this makes the whole procedure adaptive. What is more, each of these "linearized" identification subproblems is much simpler than the original off-line scheme, in which source dynamics were recovered only at the end of the whole observation horizon.

Another benefit of the proposed adaptive scheme is that it provides room for the optimization of sensor motions. Indeed, at the beginning of each stage, optimized sensor trajectories can be determined based on the predicted parameter values which are the parameter estimates coming from the previous stage. These trajectories are supposed to be specified so that the information content of the measurements with respect to the source behavior and the PDE model is as high as possible.

The problem of optimal sensor guidance for parameter estimation in DPSs has been extensively investigated over the past twenty years, but the corresponding methods have never been exploited in the context of the source identification problem. Therefore, one of our goals was to adapt some existing techniques to this setting. For the reader's convenience, in what follows we provide a concise overview of the relevant contributions.

1.5.3. Sensor trajectory design in DPSs: State of the art. The importance of sensor planning has already been recognized in numerous related application domains, e.g., air quality monitoring systems, groundwater-resources management, the recovery of valuable minerals and hydrocarbon, model calibration in meteorology and oceano- graphy, chemical engineering, hazardous environments and smart materials (Nehorai et al., 1995; Porat and Nehorai, 1996; Jeremić and Nehorai, 1998; Jeremić and Nehorai, 2000; Navon, 1997; Daescu and Navon, 2004; Christofides, 2001; Banks et al., 1996; Sun, 1994; Uciński, 2005). The operation and control of such systems usually require precise information on the parameters which condition the accuracy of the underlying mathematical model, but that information is only available through a limited number of possibly expensive sensors. Over the past years, this limitation has stimulated laborious research on the development of strategies for efficient sensor placement (for reviews, see the papers by Kubrusly and Malebranche (1985), van de Wal and de Jager (2001), and the comprehensive monographs by Uciński (1999; 2005)). Nevertheless, although the need for systematic methods was widely recognized, most techniques communicated by various authors usually are limited to sensor network nodes with no mobility, and rely on exhaustive search over a predefined set of candidates. What is more, the combinatorial nature of the design problem is taken into account very occasionally (van de Wal and de Jager, 2001). Needless to say, this approach, which is feasible for a relatively small number of possible locations, soon becomes useless as the number of possible location candidates increases or the nodes are mobile.

In the context of parameter estimation, exceptions to this naive approach constitute the works originating in statistical optimum experimental design (Fedorov and Hackl, 1997; Walter and Pronzato, 1997; Uciński and Bogacka, 2005; Uciński and Atkinson, 2004), and its extensions to models for dynamic systems, especially in the context of the optimal choice of sampling instants and input signals (Ljung, 1999; Gevers, 2005; Hjalmarsson, 2005). In this vein, various computational schemes have been developed to directly attack the original problem or its convenient approximation. The adopted optimization criteria are essentially the same, i.e., various scalar measures of performance based on the Fisher information matrix (FIM) associated with the parameters to be identified are maximized. The underlying idea is to express the goodness of parameter estimates in terms of the covariance matrix of the estimates. For sensorlocation purposes, one assumes that an unbiased and efficient (or minimum-variance) estimator is employed so that the optimal sensor placement can be determined independently of the estimator-used. This leads to a great simplification since the Cramér-Rao lower bound for the aforementioned covariance matrix is merely the inverse of the FIM, which can be computed with relative ease, even though the exact covariance matrix of a particular estimator is very difficult to obtain.

The first treatments of this type for the sensorlocation problem were proposed by Uspenskii and Fedorov (1975) as well as Rafajłowicz (1981), cf. also another 
work of Rafajłowicz (1983). Over the past two decades, this methodology has been substantially refined to extend its applicability. A comprehensive treatment of both theoretical and algorithmic aspects of the resulting sensor location strategies is contained in the monograph by Uciński (2005). The potential of the approach for generalizations was exploited, e.g., in the work by Uciński and Patan (2007), describing the setting in which a large number of possible sites at which to locate a sensor are given, but cost constraints allow only some proper subset of them to be selected. The search for the optimal solution was performed using the branch-and-bound method in which an extremely simple and efficient technique was employed to produce an upper bound to the maximum objective function.

Using mobile sensor network nodes, we can expect the minimal value of an adopted design criterion to be lower than the one with no mobility. In the seminal article by Rafajłowicz (1986), the D-optimality criterion is considered and an optimal time-dependent measure is sought, rather than the trajectories themselves. On the other hand, in the works by Uciński (2005; 2000a; 2000b) as well as Uciński and Korbicz (2001), apart from generalizations, some computational algorithms are developed based on the FIM. The problem is reduced to a state-constrained optimal-control one for which solutions are obtained via the method of successive linearizations, which is capable of handling various constraints imposed on sensor motions. In turn, Uciński and Chen (2005) attempted to properly formulate and solve the time-optimal problem for moving sensors which observe the state of a DPS so as to estimate some of its parameters. In the paper by Uciński and Chen (2006), a similar technique was presented so as to make the Hessian of the parameter estimation cost well conditioned subject an additional constraint imposed on the achievable D-efficiency of the solutions. This line of research was extended in the monograph by Song et al. (2009) towards applications involving sensor networks.

Finally, we should also pass a reference to the works by Demetriou (2006a; 2006b; 2007; 2009), Demetriou and Hussein (2009), Hussein and Demetriou (2007), as well as Uciński and Demetriou (2008) focused on on-line optimal guidance of actuator/sensor network nodes for control and/or state estimation, which demonstrate that the inclusion of a DPS model into the optimization setting can substantially improve the quality of network performance.

\subsubsection{Brief outline of the proposed sensor routing.} We assume that the original observation horizon is arbitrarily partitioned into a finite sequence of consecutive subintervals which will be further called stages. In much the same way as in the work of Uciński (2005, Chapter 4), at the beginning of each stage, the expected estimation accuracy is quantified based on a scalar measure defined on the Fisher information matrix related to the unknown parame- ters (they can characterize both the sources and the relevant PDE). The sensor routing problem over the current stage is then formulated as an optimal-control one with state-variable inequality constraints representing geometric constraints induced by the admissible measurement regions and allowable distances between the sensor nodes. Taking account of the dynamic models of the vehicles conveying the sensors, the problem is finally reduced to the determination of the control forces exerting on the sensor nodes. After a transformation of the resulting optimal control problem to the canonical Mayer form, its numerical solution can be determined using common computational tools for algorithmic optimal control, e.g., RIOTS_95.

\section{Source identification procedure}

2.1. Optimum source identification problem. Consider a (possibly nonlinear) DPS

$$
\frac{\partial y}{\partial t}=\mathcal{A}(x, t, y)+f(x, t), \quad(x, t) \in \Omega \times T
$$

with initial and boundary conditions of the general form

$$
\begin{aligned}
y(x, 0) & =0, & x & \in \Omega, \\
\mathcal{B}(x, t, y) & =0, & (x, t) & \in \Gamma \times T,
\end{aligned}
$$

where $\Omega \subset \mathbb{R}^{2}$ is a fixed, bounded, open set with sufficiently smooth boundary $\Gamma$, the points of which will be denoted by $x=\left(x_{1}, x_{2}\right), \mathcal{A}$ is a (possibly nonlinear) spatial differential operator which includes first- and secondorder spatial derivatives, $\mathcal{B}$ is an operator acting on $\Gamma, f$ signifies a source term, $t$ stands for time, $T=\left(0, t_{f}\right)$, $y=y(x, t)$ signifies the state variable with values in $\mathbb{R}$.

We assume that, for a given set of initial and boundary conditions and for each appropriate function $f$, the system of equations (1)-(3) has a unique solution. The mathematically delicate questions of the existence, uniqueness and smoothness of solutions to Eqn. (1) are beyond the scope of this work.

In the treatment to follow, we shall assume that the source term constitutes a sum of contributions coming from $r$ sources, and each of them may be decomposed into the product of a temporally dependent concentration $w_{i} \geq 0$ and a function $g_{i} \geq 0$ defining the spatial distribution of the source, such that

$$
f(x, t)=\sum_{i=1}^{r} g_{i}\left(x-\xi_{i}(t)\right) w_{i}(t),
$$

where a continuous function $\xi_{i}:\left[0, t_{f}\right] \rightarrow \bar{\Omega}=\Omega \cup \Gamma$ defines the trajectory of the $i$-th source. As for possible options for $g_{i}$, we might be given, e.g.,

$$
g_{i}(x)=\delta(x), \quad i=1, \ldots, r
$$


$\delta$ being the Dirac delta function concentrated at the origin, which correspond to pointwise sources, or Gaussian models

$$
g_{i}(x)=\exp \left(-\beta_{i}\|x\|^{2}\right), \quad i=1, \ldots, r,
$$

where $\beta_{i}>0$ are constants. In turn, as for options for $w_{i}$, unless otherwise stated, we assume that

$$
w_{i}(t)=\varrho_{i}\left(H_{b_{i}}(t)-H_{a_{i}}(t)\right),
$$

where $0 \leq a_{i}<b_{i}, \varrho_{i} \geq 0$ and $H_{\tau}(t)$ denotes the Heaviside function

$$
H_{\tau}(t)= \begin{cases}0 & \text { if } t<\tau \\ 1 & \text { otherwise }\end{cases}
$$

This means that each source releases a substance at a constant rate over the time interval $\left[a_{i}, b_{i}\right)$.

Note that in our approach the number of sensors is supposed to be known in advance.

Assume that the state of the DPS in question can be measured by a set of $N$ mobile sensor network nodes indexed by $j=1, \ldots, N$. Let $x^{j}: T \longrightarrow \Omega_{\text {ad }}$ be the trajectory of the $j$-th sensor, where $\Omega_{\mathrm{ad}} \subset \bar{\Omega}$ stands for the region where measurements can be made. The observations are assumed to be of the form

$$
z(t)=y_{\mathrm{m}}(t)+\varepsilon_{\mathrm{m}}(t), \quad t \in T,
$$

where

$$
\begin{aligned}
& y_{\mathrm{m}}(t)=\operatorname{col}\left[y\left(x^{1}(t), t\right), \ldots, y\left(x^{N}(t), t\right)\right], \\
& \varepsilon_{\mathrm{m}}(t)=\operatorname{col}\left[\varepsilon\left(x^{1}(t), t\right), \ldots, \varepsilon\left(x^{N}(t), t\right)\right],
\end{aligned}
$$

$z(t)$ is the $N$-dimensional observation vector and $\varepsilon=$ $\varepsilon(x, t)$ is a white Gaussian noise process (a formal time derivative of a Wiener process) whose statistics are

$$
\begin{aligned}
\mathrm{E}\{\varepsilon(x, t)\} & =0, \\
\mathrm{E}\left\{\varepsilon(x, t) \varepsilon\left(x^{\prime}, t^{\prime}\right)\right\} & =\sigma^{2} \delta\left(x-x^{\prime}\right) \delta\left(t-t^{\prime}\right),
\end{aligned}
$$

$\sigma>0$ being the standard deviation of the measurement noise. The assumption that we are in a position to observe directly the system state is made only for simplicity of presentation. The approach outlined in what follows can be easily generalized to indirect observation of state variables.

A preliminary version of our source identification problem is formulated as follows: We seek the source trajectories $\xi_{i}$ and intensities $w_{i}$ that yield a simulated concentration field which best matches the data from the sensors, i.e., the ones that minimize the mismatch functional

$$
\mathcal{J}(\xi, w)=\frac{1}{2} \sum_{j=1}^{N} \int_{T}\left\|z(t)-\hat{y}_{\mathrm{m}}(t ; \xi, w)\right\|^{2} \mathrm{~d} t,
$$

where

$$
\begin{aligned}
& \hat{y}_{\mathrm{m}}(t ; \xi, w) \\
& \quad=\operatorname{col}\left[\hat{y}\left(x^{1}(t), t ; \xi, w\right), \ldots, \hat{y}\left(x^{N}(t), t ; \xi, w\right)\right]
\end{aligned}
$$

and $\hat{y}(\cdot, \cdot ; \xi, w)$ denotes the solution to Eqns. (1)- (3) corresponding to given functional parameters $(\xi, w)=$ $\left(\xi_{1}, \ldots, \xi_{r}, w_{1}, \ldots, w_{r}\right)$.

Note that the above estimation process is usually ill posed in the sense that noise in the data may give rise to significant errors in the estimate. Accordingly, a regularization functional is then usually added to (12) to impose stability or a priori information, or both (Vogel, 2002).

Before proceeding with solution, we need to address the issue of reducing the problem of source identification to a finite-dimensional form. As Zhao and Nehorai(2006) did, here we apply the parametrization approach commonly used in algorithmic optimal control (Polak, 1997; Pytlak, 1999). It corresponds to explicit discretization of the source trajectories $\xi_{i}$. We can represent them, e.g., as linear combinations of canonical Lagrange interpolation polynomials or B-splines. To a great extent, the limitation of admissible source trajectories to a finite-dimensional subspace becomes a necessity if we wish to make the source identification problem tractable. Clearly, the selection of the subspace affects both the accuracy of numerical integration and the accuracy with which the solutions of the original problem are approximated. But a thorough analysis of this choice falls far beyond the scope of this paper. The reader interested in this complex problem is referred to specialized literature (e.g., Polak, 1997; Schwartz et al, 1997).From now on we thus make the assumption that the source trajectories can be represented as parametric curves of the form

$$
\xi_{i}(t)=\eta\left(t, \alpha_{i}\right), \quad t \in T,
$$

where $\eta$ denotes a given function such that $\eta\left(\cdot, \alpha_{i}\right)$ is continuous for each fixed parameter vector $\alpha_{i} \in A \subset \mathbb{R}^{p}, A$ being a given compact set. For example, if the $i$-th source sets off from the initial position at point $x_{0}^{i}=\left(x_{01}, x_{02}\right) \in$ $\bar{\Omega} \subset \mathbb{R}^{2}$ and moves along a straight line with constant velocity $v^{i}=\left(v_{1}^{i}, v_{2}^{i}\right) \in \mathbb{R}^{2}$, then its parameterized path model is

$$
\eta\left(t, \alpha_{i}\right)=x_{0}^{i}+v^{i} t,
$$

where $\alpha_{i}=\left(x_{01}, x_{02}, v_{1}^{i}, v_{2}^{i}\right)$

According to the above remarks, in order to model the action of the $i$-th source, we can form a vector $\theta^{i}=$ $\left(\varrho_{i}, a_{i}, b_{i}, \alpha_{i}, \beta_{i}\right)$ which consists of constant parameters. In consequence, the cumulative effect of all sources will dictated by the parameter vector

$$
\theta=\left(\theta^{1}, \ldots, \theta^{r}\right)
$$

Consequently, our modified form of the source identification problem then consists in minimizing the fit-to-data 
criterion

$$
\mathcal{J}(\theta)=\frac{1}{2} \int_{T}\left\|z(t)-\hat{y}_{\mathrm{m}}(t ; \theta)\right\|^{2} \mathrm{~d} t,
$$

where

$$
\hat{y}_{\mathrm{m}}(t ; \theta)=\operatorname{col}\left[\hat{y}\left(x^{1}(t), t ; \theta\right), \ldots, \hat{y}\left(x^{N}(t), t ; \theta\right)\right]
$$

and $\hat{y}(\cdot, \cdot ; \theta)$ stands for the solution to (1)-(3) corresponding to a given vector of parameters $\theta$ specifying the source term through (4), (7) and (14).

We feel, intuitively, that the parameter estimate $\widehat{\theta}$ depends on the trajectories $x^{j}$ since the integrand on the right-hand side of Eqn. (17) does. This fact suggests that we may attempt to select sensor trajectories which would lead to the best estimates of the system parameters. To form a basis for the comparison of different trajectories, a quantitative measure of the 'goodness' of particular trajectories is required. A logical approach is to choose a measure related to the expected accuracy of the parameter estimates to be obtained from the data collected (note that the design is to be performed off-line, before taking any measurements). Such a measure is usually based on the concept of the Fisher Information Matrix (FIM), (Sun, 1994; Rafajłowicz, 1986), which is widely used in optimum experimental design theory for lumped systems (Walter and Pronzato, 1997; Fedorov and Hackl, 1997; Atkinson et al., 2007). When the time horizon is large, the nonlinearity of the model with respect to its parameters is mild and the measurement errors are independently distributed and have small magnitudes, the inverse of the FIM constitutes a good approximation of the covariance matrix for the estimate of $\theta$ (Walter and Pronzato, 1997; Fedorov and Hackl, 1997; Atkinson et al., 2007).

For notational convenience, introduce

$$
s(t)=\left(x^{1}(t), x^{2}(t), \ldots, x^{N}(t)\right), \quad \forall t \in T
$$

and set $n=\operatorname{dim}(s(t))$. The FIM has the following representation (Uciński, 2005; Quereshi et al., 1980):

$$
M(s)=\sum_{j=1}^{N} \int_{0}^{t_{f}} g\left(x^{j}(t), t\right) g^{\top}\left(x^{j}(t), t\right) \mathrm{d} t,
$$

where

$$
g(x, t)=\left.\nabla_{\theta} y(x, t ; \theta)\right|_{\theta=\theta^{0}}
$$

denotes the vector of the so-called sensitivity coefficients, $\theta^{0}$ being a prior estimate to the unknown parameter vector $\theta$ (Uciński, 2000b; Uciński, 2005).

Optimal sensor trajectories can be found by choosing $s$ so as to maximize some scalar function $\Psi$ of the information matrix. The introduction of the design criterion permits to cast the sensor location problem as an optimization one, and the criterion itself can be treated as a measure of the information content of the observations. Several choices exist for such a function (cf. Walter and Pronzato, 1997; Fedorov and Hackl 1997; Atkinson et al., 2007), and the most popular one is the D-optimality criterion:

$$
\Psi[M]=\log \operatorname{det}(M) .
$$

Its use yields the minimal volume of the confidence ellipsoid for the estimates.

\subsection{Limitations on sensor movements.}

2.2.1. Dynamics. We assume that the sensors are conveyed by vehicles whose motions are described by

$$
\dot{s}(t)=h(s(t), u(t)) \quad \text { a.e. on } T, \quad s(0)=s_{0},
$$

where a given function $h: \mathbb{R}^{n} \times \mathbb{R}^{p} \rightarrow \mathbb{R}^{n}$ is required to be continuously differentiable, $s_{0} \in \mathbb{R}^{n}$ defines an initial sensor configuration, and $u: T \rightarrow \mathbb{R}^{p}$ is a measurable control function which satisfies

$$
u_{l} \leq u(t) \leq u_{u} \quad \text { a.e. on } T
$$

for some constant vectors $u_{l}$ and $u_{u}$.

Given any initial sensor configuration $s_{0}$ and any control function, there is a unique absolutely continuous function $s: T \rightarrow \mathbb{R}^{n}$ which satisfies (23) a.e. on $T$. In what follows, we will call it the state trajectory corresponding to $s_{0}$ and $u$, and make the following notational convention: if $s$ appears without mention in a formula, it is always understood that a control $u$ and initial condition $s_{0}$ have been specified while $s$ is the trajectory corresponding to $u$ and $s_{0}$ through (23).

2.2.2. Pathwise state constraints. In reality, some restrictions on the motions are inevitably induced. First of all, all sensor network nodes should stay within the admissible region $\Omega_{\mathrm{ad}}$ where measurements are allowed. We assume that it is a compact set defined as follows:

$$
\Omega_{\mathrm{ad}}=\left\{x \in \bar{\Omega}: b_{i}(x) \leq 0, i=1, \ldots, I\right\},
$$

where $b_{i}$ 's are given continuously differentiable functions Accordingly, the conditions

$$
\lambda_{i j}(s(t))=b_{i}\left(x^{j}(t)\right) \leq 0, \quad \forall t \in T,
$$

must be fulfilled, where $1 \leq i \leq I$ and $1 \leq j \leq N$.

Moreover, we can restrict the admissible distances between the sensors by imposing the constraints

$$
\mu_{i j}(s(t))=R^{2}-\left\|x^{i}(t)-x^{j}(t)\right\|^{2} \leq 0, \quad \forall t \in T,
$$

where $1 \leq i<j \leq N$ and $R$ stands for a minimum allowable distance which guarantees that the measurements taken by the sensors can be considered independent. 
To shorten the notation, after relabelling, we rewrite constraints 26) and 27) in the form

$$
\gamma_{\ell}(s(t)) \leq 0, \quad \forall t \in T
$$

where $\gamma_{\ell}, \ell=1, \ldots, I N$ tally with (26), whereas $\gamma_{\ell}, \ell=$ $I N+1, \ldots,[I+(N-1) / 2] N$ coincide with 27). In the sequel, $\bar{\nu}$ stands for the set if indices $\{1, \ldots, \nu\}, \nu=$ $[I+(N-1) / 2] N$.

2.3. Optimal control formulation. The goal in the optimal measurement problem is to determine the forces (controls) applied to each vehicle conveying a sensor, which maximize the design criterion $\Psi[\cdot]$ defined on the FIMs of the form (20), which are determined unequivocally by the corresponding trajectories, subject to the constraints (24) on the magnitude of the controls and induced state constraints (28). In order to increase the degree of optimality, in our approach we will regard $s_{0}$ as a control parameter vector to be chosen in addition to the control function $u$. Clearly, the correctness of such a formulation necessitates some additional restrictions on the smoothness of sensitivity coefficients $g$. In what follows, we thus assume the continuity of $g$ and $\partial g / \partial x$.

The above formulation can be interpreted as the following optimization problem: Find the pair $\left(s_{0}, u\right)$ which maximizes

$$
J\left(s_{0}, u\right)=\Psi[M(s)]
$$

over the set of feasible pairs

$$
\begin{aligned}
\mathcal{P}=\left\{\left(s_{0}, u\right) \mid u: T \rightarrow \mathbb{R}^{p}\right. \text { is measurable, } \\
\left.u_{l} \leq u(t) \leq u_{u} \text { a.e. on } T, s_{0} \in \Omega_{\mathrm{ad}}^{N}\right\},
\end{aligned}
$$

subject to the pathwise state inequality constraints (28).

Evidently, its high non-linearity excludes any possibility of finding closed-form formulas for its solution. Accordingly, we must resort to numerical techniques. A number of possibilities exist in this respect (Polak, 1997; Gruver and Sachs, 1980), but before exploiting them, observe that in spite of its apparently non-classical form, the resulting optimal-control problem can be easily cast as a classical Mayer problem where the performance index is defined only via terminal values of state variables.

2.4. Equivalent canonical problem. The aim of this section is to convert our problem into a canonical optimal control one with an endpoint cost and inequalityconstrained trajectories (Polak, 1997). Such a transcription will make it possible to employ existing software packages for numerical solving of dynamic optimization and optimal control problems.

For notational convenience, define the function svec : $\mathbb{S}^{m} \rightarrow \mathbb{R}^{m(m+1) / 2}$, where $\mathbb{S}^{m}$ denotes the subspace of all symmetric matrices in $\mathbb{R}^{m \times m}$, that takes the lower triangular part (the elements only on the main diagonal and below) of a symmetric matrix $A$ and stacks them into a vector $a$ :

$$
\begin{aligned}
a= & \operatorname{svec}(A) \\
= & \operatorname{col}\left[A_{11}, A_{21}, \ldots, A_{m 1}, A_{22},\right. \\
& \left.A_{32}, \ldots, A_{m 2}, \ldots, A_{m m}\right] .
\end{aligned}
$$

Similarly, let $A=\operatorname{Smat}(a)$ be the symmetric matrix such that $\operatorname{svec}(\operatorname{Smat}(a))=a$ for any $a \in \mathbb{R}^{m(m+1) / 2}$.

Consider the matrix-valued function

$$
\Pi(s(t), t)=\sum_{j=1}^{N} g\left(x^{j}(t), t\right) g^{\top}\left(x^{j}(t), t\right) .
$$

Setting $\zeta: T \rightarrow \mathbb{R}^{m(m+1) / 2}$ as the solution of the differential equations

$$
\dot{\zeta}(t)=\operatorname{svec}(\Pi(s(t), t)), \quad r(0)=0,
$$

we have

$$
M(s)=\operatorname{Smat}\left(\zeta\left(t_{f}\right)\right)
$$

i.e., the maximization of $\Psi[M(s)]$ thus reduces to the maximization of a function of the terminal value of the solution to 33 .

Introducing the augmented state vector

$$
q(t)=\left[\begin{array}{l}
s(t) \\
\zeta(t)
\end{array}\right]
$$

we obtain

$$
q_{0}=q(0)=\left[\begin{array}{c}
s_{0} \\
0
\end{array}\right] .
$$

Then the equivalent canonical optimal control problem consists in finding a pair $\left(q_{0}, u\right) \in \overline{\mathcal{P}}$ which maximizes the performance index

$$
\bar{J}\left(q_{0}, u\right)=\psi\left(q\left(t_{f}\right)\right)
$$

subject to

$$
\left\{\begin{aligned}
\dot{q}(t) & =\varphi(q(t), u(t), t) \\
q(0) & =q_{0}, \\
\bar{\gamma}_{\ell}(q(t)) & \leq 0, \quad \forall t \in T, \quad \ell \in \bar{\nu},
\end{aligned}\right.
$$

where

$$
\begin{aligned}
\overline{\mathcal{P}}=\left\{\left(q_{0}, u\right) \mid u: T \rightarrow \mathbb{R}^{r}\right. \text { is measurable } \\
\left.u_{l} \leq u(t) \leq u_{u} \text { a.e. on } T, s_{0} \in \Omega_{\mathrm{ad}}^{N}\right\}
\end{aligned}
$$

and

$$
\begin{aligned}
\varphi(q, u, t) & =\left[\begin{array}{c}
h(s(t), u(t)) \\
\operatorname{svec}(\Pi(s(t), t))
\end{array}\right], \\
\psi(q(t)) & =\Psi[\operatorname{Smat}(\zeta(t))], \\
\bar{\gamma}_{\ell}(q(t)) & =\gamma_{\ell}(s(t)) .
\end{aligned}
$$


The above problem in canonical form can be solved using one of the existing packages for numerical solving dynamic optimization problems, such as RIOTS_95 (Schwartz et al., 1997), DIRCOL (von Stryk, 1999), or MISER (Jennings et al., 2002). In our implementation, we employed the first one, i.e., RIOTS_95, which is designed as a MATLAB toolbox written mostly in $\mathrm{C}$, and runs under Windows 98/2000/XP and Linux. It provides an interactive environment for solving a very broad class of optimal control problems. Users' problems can be prepared purely as M-files and no compiler is required to solve them. To speed up the solution process, the functions defining the problem can be coded in $\mathrm{C}$ and then compiled and linked with some pre-built linking libraries. The implemented numerical methods are supported by the theory outlined by Polak (1997), which uses the approach of consistent approximations. Systems dynamics can be integrated with the fixed step-size Runge-Kutta integration, a discrete-time solver or a variable step-size method. The software automatically computes gradients for all functions with respect to the controls and any free initial conditions. The controls are represented as splines, which allows a high-degree of function approximation accuracy without requiring a large number of control parameters. There are three main optimization routines, each suited for different levels of generality, and the most general one is based on sequential quadratic programming methods (it was also used in our computations reported in Section 3 ).

\subsection{Adaptive trajectory planning based on sequential designs.}

2.5.1. General idea. One of the main difficulties associated with the optimization of sensor locations is the dependence of optimal solutions on the true values $\theta_{\text {true }}$ of the parameters to be estimated (Uciński, 2005, p. 22). Since these values are unknown, an obvious and common approach is to construct locally optimal designs described in the previous sections for some prior estimate $\theta^{0}$ of $\theta_{\text {true }}$ in lieu of $\theta_{\text {true }}$ itself, cf. the definition of the sensitivity coefficient (21). It can be, e.g., a nominal value for $\theta$ or a result of a preliminary experiment. But $\theta^{0}$ may be far from $\theta_{\text {true }}$ and, simultaneously, properties of locally optimal designs can be very sensitive to changes in $\theta$ (Ford et al., 1989). Such prior uncertainty on $\theta^{0}$ is not taken into account by any optimization procedure to determine local designs, and an experimental setting thus obtained may consequently be far from optimal.

A very natural idea is to alternate experimentation and estimation steps. Accordingly, the total time horizon is divided into several contiguous parts and each of them is related to the corresponding stage of the experiment. At each stage, in turn, locally optimal sensor trajectories are determined based on the available parameter estima-

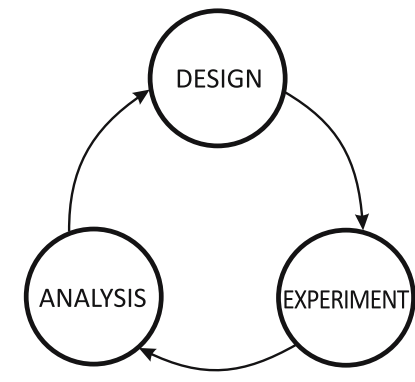

Fig. 1. General scheme of adaptive sequential design.

tes (nominal parameter values can be assumed as initial guesses for the first stage), measurements are taken at the newly calculated sensor positions, and the data obtained are then analyzed and used to update the parameter estimates (see Fig. 1). In this general scheme, it is intuitively supposed that each estimation phase improves our knowledge about the parameters and this knowledge can then be used to improve the quality of the next experiment to be performed.

Owing to its simplicity, sequential design is commonly considered a universal panaceum for the shortcomings of local designs. Let us note, however, that the following important questions are to be faced (Ford et al., 1989), and the answers to them are by no means straightforward:

1. How many subintervals should be chosen?

2. How do the initial estimates of parameters influence the design?

3. What are the asymptotic properties of sequential procedures, i.e., does the generated design 'tend' in any sense to a design which would be optimal in terms of the true $\theta$ ?

Some developments regarding a theoretical justification for the sequential approach and its convergence properties can be found, e.g., in the works of Ford et al. (1989) or Walter and Pronzato (1990; 1997). Sequential designs have been employed in our work to incorporate the hitherto collected measurements into the process of constructing optimized sensor node trajectories.

2.6. Reliability measure for source detection. Having outlined a general idea of model-based source identification, we now turn our attention to implementation details. Assume that the sensors collect measurements of the contaminant concentration at discrete time moments $t_{q}=q \Delta t$, i.e., the observations made by the $j$-th sensor are of the form

$$
\begin{aligned}
& z^{j}\left(t_{q}\right)=y\left(x^{j}\left(t_{q}\right), t_{q} ; \theta\right)+\varepsilon^{j}\left(x^{j}\left(t_{q}\right), t_{q}\right), \\
& j=1, \ldots, N, \quad q=1, \ldots, Q_{T},
\end{aligned}
$$


where $\varepsilon^{j}\left(x^{j}\left(t_{q}\right), t_{q}\right)$ can be interpreted as realizations of independent, identically distributed Gaussian random varables with zero mean and variance $\sigma^{2}$. Accordingly, the estimate $\hat{\theta}$ of the unknown parameter vector $\theta$ is then determined as a global minimizer of the least-squares criterion

$$
\mathcal{J}(\vartheta)=\sum_{j=1}^{N} \sum_{q=1}^{Q_{T}}\left[z^{j}\left(t_{q}\right)-\hat{y}\left(x^{j}\left(t_{q}\right), t_{q} ; \vartheta\right)\right]^{2},
$$

where $\hat{y}(\cdot, \cdot ; \vartheta)$ denotes the solution of (1)-(3) for a given value of the parameter vector $\vartheta$.

An elementary idea of source detection is to compare the resulting parameter estimates with the corresponding known nominal values, treating possible differences as residuals which contain information about potential contamination sources. Based on some thresholding techniques, the appropriate decision making system could be constructed to detect abnormal situations in system functioning (Patan and Uciński, 2005; 2008; Patan and Patan, 2005).

Basically, only a subset of system parameters describes the properties of contamination sources. Furthermore, only some of the parameters characterizing the source are useful for source detection. This accounts for partitioning the parameter vector into two subsets. With no loss of generality, we may write

$$
\theta=\left[\begin{array}{lll:lll}
\theta_{1} & \ldots & \theta_{\ell} & \theta_{\ell+1} & \ldots & \theta_{m}
\end{array}\right]^{\top}=\left[\begin{array}{l:l}
\alpha^{\top} & \beta^{\top}
\end{array}\right]
$$

where $\alpha$ is a vector of $\ell$ parameters which are essential for a proper source detection and/or localization. Vector $\beta$ contains some unknown parameters which are an important part of the model but are useless for detection (or localization in a further stage). Based on the observations, it is possible to test the simple null hypothesis

$$
H_{0}: \alpha=\alpha^{0}
$$

where $\alpha^{0}$ is the nominal value for the vector $\alpha$ corresponding to the system performance when the contamination source is absent.

The generalization of the log-likelihood function for the experiment considered takes the following form (Goodwin and Payne, 1977):

$$
\begin{aligned}
\mathcal{L}(z ; \theta)= & \ln \left(2 \pi \sigma^{2}\right)^{-Q_{T} N / 2} \\
& -\frac{1}{2 \sigma^{2}} \sum_{j=1}^{N} \sum_{q=1}^{Q_{T}}\left|z^{j}\left(t_{q}\right)-\hat{y}\left(x^{j}\left(t_{q}\right), t_{q} ; \theta\right)\right|^{2} .
\end{aligned}
$$

Setting $\Theta_{0}=\left\{\theta \in \Theta: \alpha=\alpha_{0}\right\}$, we can define the following generalized log-likelihood ratio:

$$
\lambda(z)=\frac{\sup _{\vartheta \in \Theta} \mathcal{L}(z ; \vartheta)}{\sup _{\vartheta \in \Theta_{0}} \mathcal{L}(z ; \vartheta)}=\frac{1}{2 \sigma^{2}}(J(\tilde{\theta})-J(\hat{\theta})),
$$

where

$$
\hat{\theta}=\arg \min _{\vartheta \in \Theta} J(\vartheta), \quad \tilde{\theta}=\arg \min _{\vartheta \in \Theta_{0}} J(\vartheta) .
$$

The likelihood ratio test is widely used in statistics. This is because even in the cases where a theoretical justification of its optimality is missing the likelihood ratio can still be shown to be very good in practice (Lehmann and Romano, 2005). Furthermore, it can be shown that, assuming the validity of the null hypothesis $H_{0}$, the sequence $\{2 \lambda(z)\}$ for $Q_{T} N \rightarrow \infty$ is weakly convergent to a $\chi^{2}$ random variable on $\ell$ degrees of freedom (Goodwin and Payne, 1977, Theorem 3.6.1, p. 55). The meaning of this fact is that we can compare the observed value of $2 \lambda(z)$ with some threshold $k_{\gamma}$ obtained from the cumulative $\chi^{2}$ distribution on $\ell$ degrees of freedom, where $k_{\gamma}$ is such that $100(1-\gamma) \%$ of the distribution lies to the left of $k_{\gamma}$. The decision rule for a given significance level $\gamma$, which represents a fixed range of model uncertainty, takes the following form:

$$
S=\left\{\begin{array}{lll}
\text { reject } H_{0} & \text { if } 2 \lambda(z) \geqslant k_{\gamma} & \text { (source is present), } \\
\text { accept } H_{0} & \text { if } 2 \lambda(z)<k_{\gamma} & \text { (source is absent ). }
\end{array}\right.
$$

The potential rejection of $H_{0}$ indicates an essential deviation of the vector $\alpha$ from the nominal value of this parameter and is a base for the detection of abnormal states in the system.

For a fixed significance level (i.e., fixed probability of false alarms), the power of the likelihood ratio test for the alternative hypothesis of the form $H_{1}: \alpha \neq \alpha^{0}$, i.e.,

$$
1 \text { - the probability of accepting } H_{0} \text { when } H_{1} \text { is true, }
$$

can be made large by maximizing the $D_{s}$-optimality criterion (see the work of Patan and Patan (2005) for details):

$$
\Psi_{s}[M]=\log \operatorname{det}\left[M_{\alpha \alpha}-M_{\alpha \beta} M_{\beta \beta}^{-1} M_{\alpha \beta}^{\top}\right],
$$

where $M \in \mathbb{R}^{m \times m}$ stands for the Fisher information matrix decomposed as

$$
M=\left[\begin{array}{c:c}
M_{\alpha \alpha} & M_{\alpha \beta} \\
\hdashline \cdots_{\alpha \beta} & M_{\beta \beta}
\end{array}\right]
$$

such that $M_{\alpha \alpha} \in \mathbb{R}^{\ell \times \ell}, M_{\alpha \beta} \in \mathbb{R}^{\ell \times(m-\ell)}, M_{\beta \beta} \in$ $\mathbb{R}^{(m-\ell) \times(m-\ell)}$.

In our settings, the FIM is given by

$$
M=\sum_{j=1}^{N} \sum_{q=1}^{Q_{T}} g\left(x^{j}\left(t_{q}\right), t_{q}\right) g^{\top}\left(x^{j}\left(t_{q}\right), t_{q}\right),
$$

where

$$
g(x, t)=\left(\frac{\partial y(x, t ; \vartheta)}{\partial \vartheta}\right)_{\vartheta=\theta^{0}}^{\top}
$$




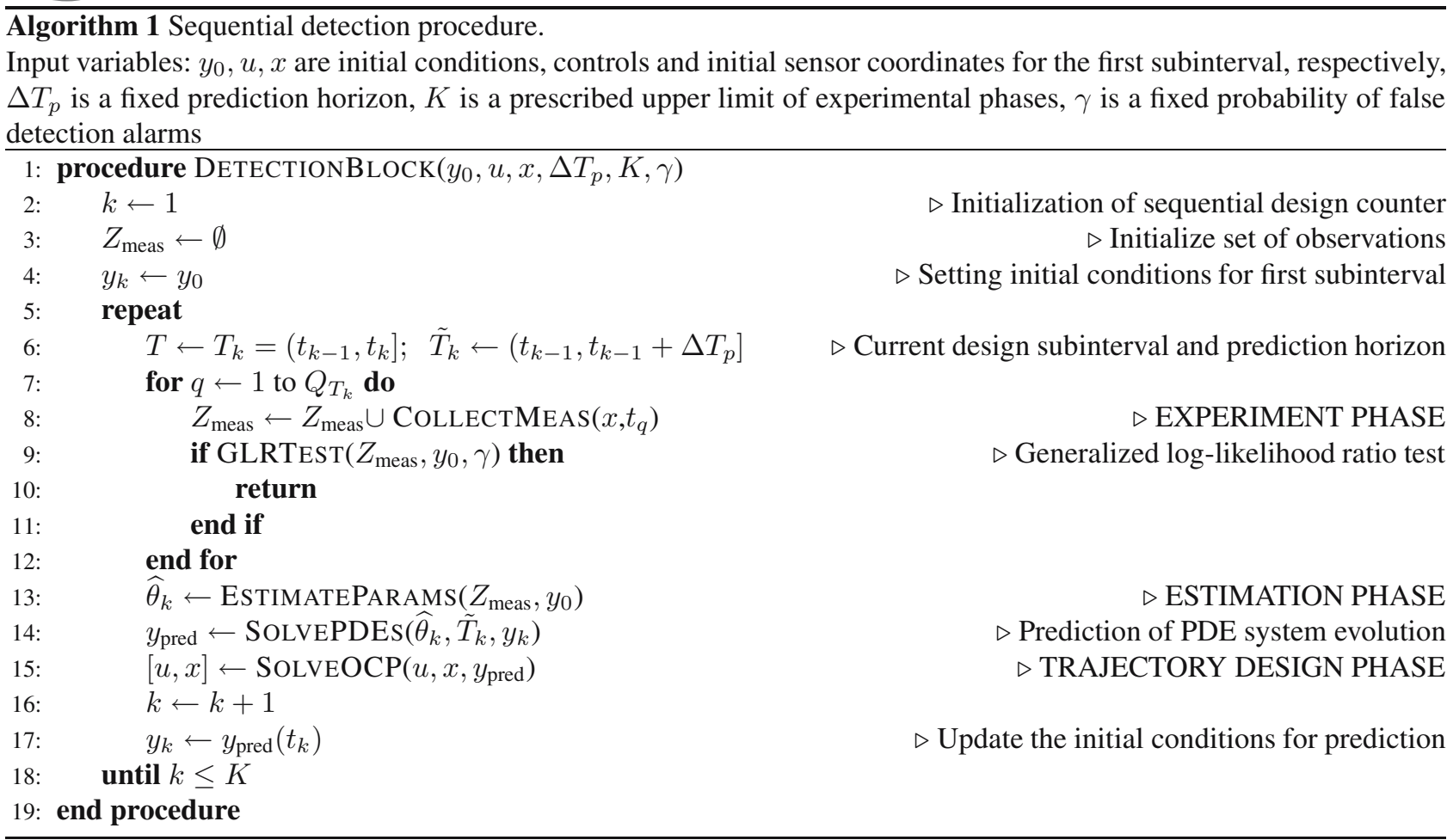

$\theta^{0}$ being a prior estimate to the unknown parameter vector $\theta$.

The trajectories providing the most informative observations for the detection and localization of a moving contamination source strongly depend on the true vector of parameters $\theta$ describing the source, which is obviously unknown. In the case when initial information about the parameters is missing, the only solution is to conduct an additional analysis for the predetermination of the initial estimates of the parameters of interest. Nevertheless, it is impossible to construct an optimal design for such an experiment without sufficient knowledge about parameters, because most often optimal sensor locations depend on $\theta$. This leads directly to a very reasonable idea of the repetition of the measurement collection, estimation, prediction and design steps several times. This is equivalent to the division of the resources (e.g., the time horizon) into small parts which are related to the corresponding consecutive stages of the experiment. More precisely, for the purpose of adaptive estimation and analysis, the total observation horizon $\mathcal{T}=\left(0, t_{f}\right]$ is divided using time moments $0=t_{0}<t_{1}<\cdots<t_{K}=t_{f}$ forming its arbitrary partition into a collection of consecutive subintervals $T_{k}=\left[t_{k-1}, t_{k}\right], k=1, \ldots, K$. Based on the observational data collected chronologically in previous subintervals, we are able to make a proper analysis and efficiently design the sensor network to update estimates of the system parameters depending on the particular purpose such as the detection or/and localization of the pollution source.
2.6.1. Detection block. An algorithmic scheme of detection block is provided in Algorithm 1 The operators involved in this implementation are as follows:

- $\operatorname{CollectMeas}\left(x, t_{q}\right)$ returns the system response measured by sensor nodes at specified time instant $t_{q}$.

- $\operatorname{GLRTest}\left(Z_{\text {meas }}, y_{0}, \gamma\right)$ is an implementation of (50). It returns true only if a log-likelihood ratio (48) calculated for the observations included in $Z_{\text {meas }}$ and initial conditions in $y_{0}$ exceeds the threshold for the probability of false alarms fixed at $\gamma$.

- EstimateParams $\left(Z_{\text {meas }}, y_{0}\right)$ determines the estimate of the system parameters for the current design interval. The GLRTEST procedure also estimates the system parameters but for detection purposes, so their accuracy is not adequate for the design purposes.

- $\operatorname{SolvEPDEs}\left(\widehat{\theta}_{k}, \tilde{T}_{k}, y_{k}\right)$ generates the forecast of the system response and its sensitivities on the interval $\tilde{T}_{k}$ based on the estimated system parameters and initial conditions $y_{k}$ estimated in the previous design interval.

- $\operatorname{SolveOCP}\left(u, x, y_{\text {pred }}\right)$ solves the corresponding optimal control problem and determines updates of the controls for sensor nodes using the predicted solution $y_{\text {pred }}$ of the PDE system.

2.6.2. Identification (localization) block. Once the source has been detected, we are able to provide a simi- 


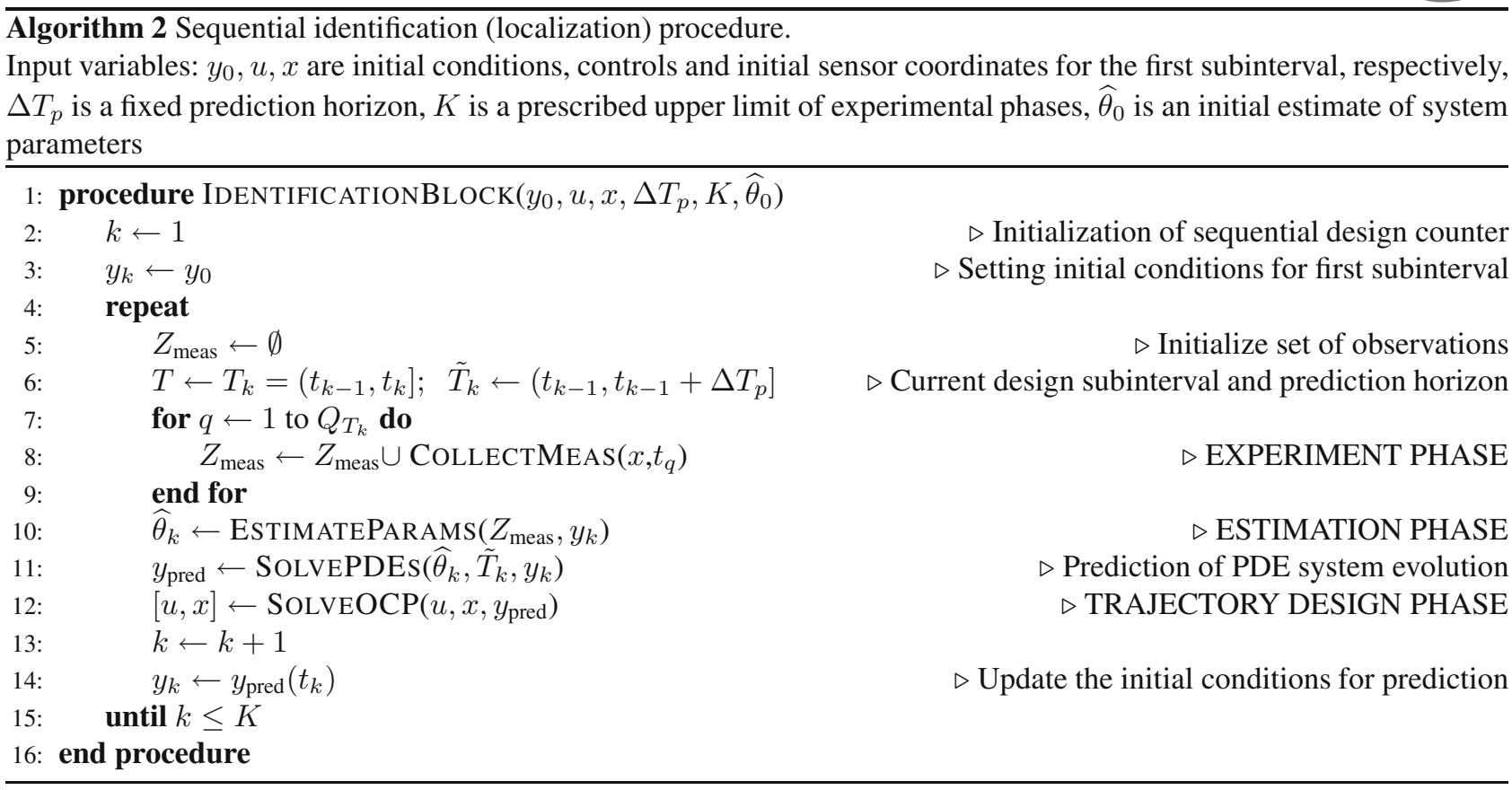

lar sequential scheme for source parameter identification starting with some parameter estimates (from the detection stage or previous experiments). A heuristic scheme of the identification block is embodied in Algorithm 2. The operators used here have the same meaning as those from the detection block.

\section{Results and discussion}

3.1. Source localization. As an illustration of the proposed approach, consider the following example, which consists in the localization of a moving contamination source within a bounded area using a sensor network with mobile nodes equipped with concentration sensors.

3.1.1. Process description. The spatial domain considered in our simulations is a square with side length of 1 $\mathrm{km}$ (see Fig. 2). An active source of a toxic pollutant moves within this domain and emits the polluting substance to the atmosphere. Emission is assumed to start at the initial time of the simulation. The size of the domain is closely related to a physical model of the mesoscale atmospheric motion (Jacobson, 1999). Therefore, the changes in the spatial concentration $y(x, t)$ of this substance over the discussed observation interval $T=[0,600]$ (in seconds) can be mathematically described by the following advectiondiffusion equation:

$$
\begin{aligned}
& \frac{\partial y(x, t)}{\partial t}+\nabla \cdot(v(x, t) y(x, t)) \\
& \quad=\nabla \cdot(\kappa \nabla y(x, t))+f(x, t), \quad(x, t) \in \Omega \times T,
\end{aligned}
$$

subject to the boundary and initial conditions:

$$
\begin{cases}\frac{\partial y(x, t)}{\partial n}=0, & (x, t) \in \Gamma \times T, \\ y(x, 0)=y_{0}, & x \in \Omega,\end{cases}
$$

where the term $f(x, t)=\mu \exp \left(-100\|x-\xi(t)\|^{2}\right)$ constitutes the model of an active source of the pollutant with the emission intensity coefficient $\mu$ and the instantaneous location given by trajectory $\xi(t)$. Furthermore, $\kappa$ denotes a turbulent diffusion coefficient and $\partial y / \partial n$ stands for the partial derivative of $y$ with respect to the outward normal to the boundary $\Gamma$. As for prior estimates of the unknown parameters $\mu$ and $\kappa$, the values of $10 \mathrm{~kg} / \mathrm{s}$ and $60 \mathrm{~m}^{2} / \mathrm{s}$ were used, respectively.

3.1.2. Experiment settings. In our simulation, the pollution source is assumed to start at $t=0$ from the point $(0.5,0.75)$, then move at a constant pace along the arc of the circle with radius $\rho=\sqrt{5} / 4$ and center at $(0.0,0.5)$, and finally terminate at point $(0.5,0.25)$ at $t=600$, i.e., its trajectory is given by

$$
\begin{aligned}
& \xi_{1}=\rho \sin \left(\frac{\omega_{1} t}{600}+\omega_{0}\right), \\
& \xi_{2}=0.5+\rho \cos \left(\frac{\omega_{1} t}{600}+\omega_{0}\right),
\end{aligned}
$$

where $\omega_{1}=2 \arcsin (0.2 \sqrt{5})$ and $\omega_{0}=\arcsin (0.4 \sqrt{5})$.

The velocity field $v(x, t)$ varies in space and time ac- 


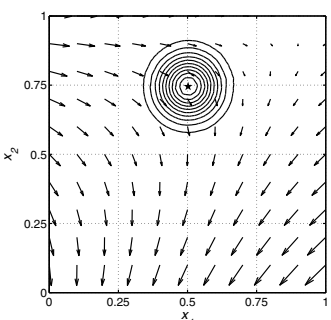

(a) $t=6 \mathrm{~s}$

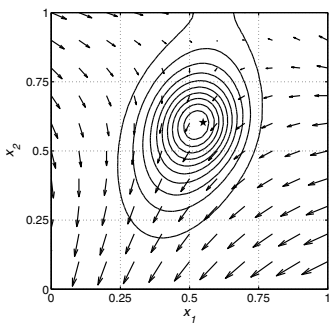

(c) $t=180 \mathrm{~s}$

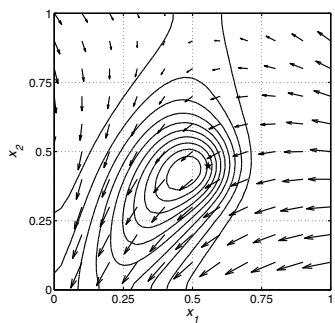

(e) $t=360 \mathrm{~s}$

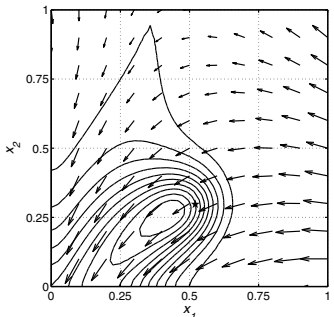

(g) $t=540 \mathrm{~s}$

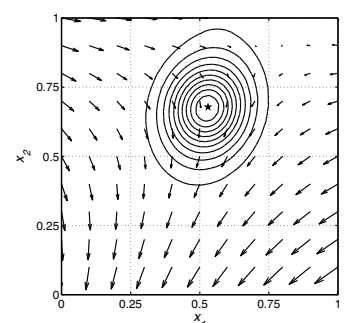

(b) $t=90 \mathrm{~s}$

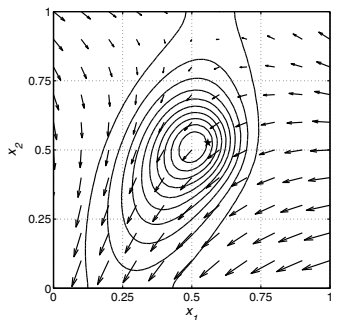

(d) $t=270 \mathrm{~s}$

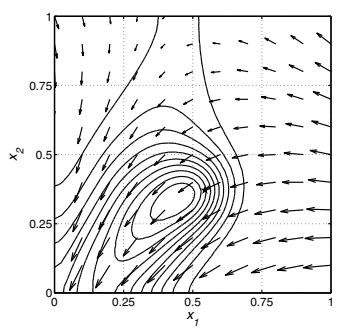

(f) $t=450 \mathrm{~s}$

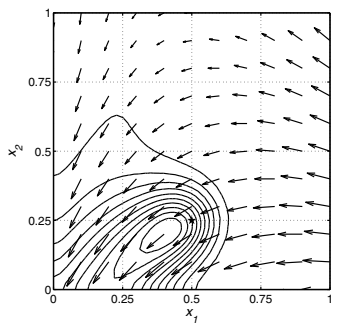

(h) $t=600 \mathrm{~s}$
Fig. 2. Evolution of the wind velocity field versus the pollutant concentration and the actual position of the pollutant source (marked with asterisks).

cording to the following model (cf. Fig. 2):

$$
\begin{aligned}
v(x, t)= & \left(12\left(x_{2}-x_{1}-\frac{t}{600}\right),\right. \\
& \left.12\left(\frac{t\left(2 x_{1}-1\right)}{600}+x_{2}-1\right)\right)[\mathrm{km} / \mathrm{h}] .
\end{aligned}
$$

The solution to 56- 57 with simulated additive measurement noise with standard deviation $\sigma=10^{-4} \mathrm{~kg} / \mathrm{m}^{3}$ constitutes the reference output signal for our simulations.

Our purpose is to determine the evolution of the source, i.e., an estimate of its trajectory $\xi(t)$ over the time interval $T$ using a sensor network with mobile nodes. Sin- ce vapor measurements cannot be taken continuously due to technical limitations, we assume that the consecutive observations are taken at discrete times every $\Delta t=15 \mathrm{~s}$. Then, we divide the observation interval into 20 equal subintervals

$$
\begin{aligned}
& T_{k}=\left(t_{k}, t_{k}+2 \Delta t\right], \quad t_{k}=2(k-1) \Delta t, \\
& k=1, \ldots, 20
\end{aligned}
$$

and approximate the source trajectory $\xi(t)$ by linear splines

$$
\xi(t) \approx \xi_{k}+v_{k}\left(t-t_{k}\right), \quad t \in T_{k}, \quad k=1, \ldots, 20,
$$

where $v_{k}=\left(x_{k+1}-x_{k}\right) /(2 \Delta t)$ is the average source velocity in the interval $T_{k}$ and $\xi_{k}$ denotes the location of the source at time $t_{k}$. The $\xi_{k}$ 's become the parameters of interest in our source localization problem.

Since, usually, it is not possible to freely deploy sensors within the domain considered, in our simulation scenario the sensors are assumed to approach the contaminated area starting from arbitrary points on the left boundary and their initial positions are fixed (i.e., they are not optimized). For the first 150 s, i.e., within the first five time subintervals, the sensors move with the maximum speed to the center of the area simultaneously collecting the measurements. Based on those observations, the first estimate of the system parameters is determined which is used as an a priori value to design the sensor trajectories for the next consecutive observation subinterval.

Then, for every $T_{k}, k=6, \ldots, 20$ we have to update the controls for the sensor network nodes in order to accurately find the current location of the source. From among the system parameters $\theta_{k}=\left(\xi_{k, 1}, \xi_{k, 2}, v_{k, 1}, v_{k, 2}, \mu, \kappa\right)$, the localization of the pollution source is based on the knowledge of the first four. Thus, the maximization of the reliability for the localization of the contamination source is equivalent to the determination of $D_{s}$-optimal sensor trajectories (Patan and Patan, 2005). In order to somewhat simplify the identification process of source coordinates and solve this problem in an on-line manner, we have applied a computational scheme similar to that used by the optimal predictive control technique. Roughly speaking, for each consecutive time subinterval $T_{k}$ the following steps are applied:

- First, the estimate $\hat{\theta}^{k}$ of the current parameter vector $\theta^{k}$ is determined based on measurements collected on subinterval $T_{k}$. The position $\xi_{k}$ is updated and stored as the source position at time $t_{k}$ and $v_{k}$ is used for the prediction of the source location and initial conditions for the system state at the next time instant $t_{k+1}$.

- The model equations (56) and (57) are solved using (61) and the current vector $\hat{\theta}^{k}$ on the interval $\tilde{T}_{k}=$ 


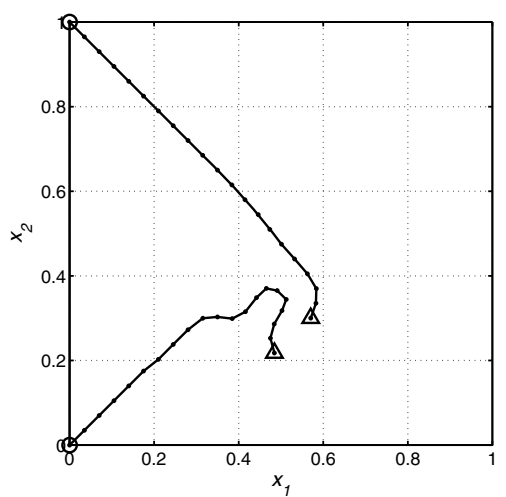

(a)

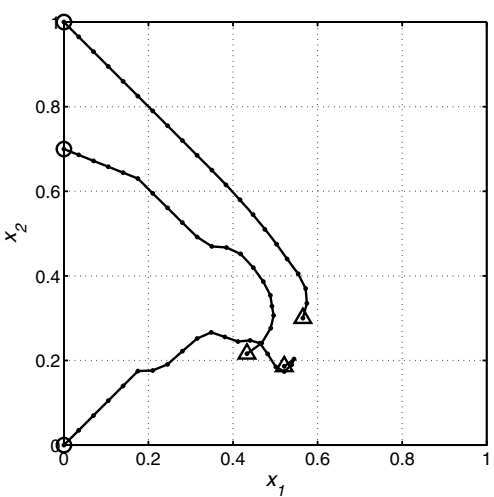

(b)

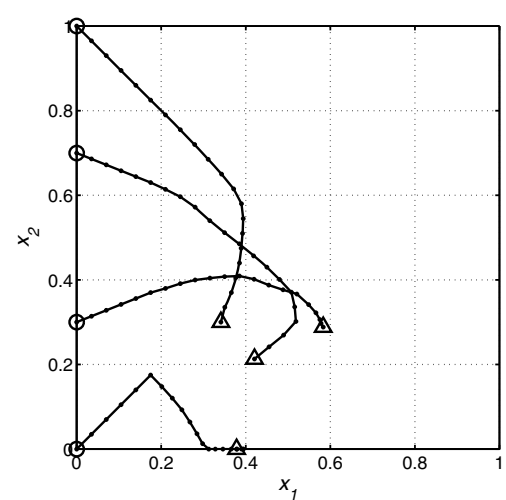

(c)

Fig. 3. Optimal sensor trajectories (starting and final positions are marked with open circles and triangles, respectively) for two (a), three (b) and four (c) sensors.

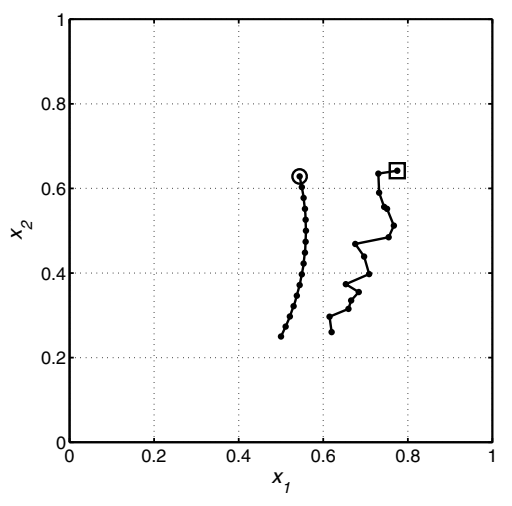

(a)

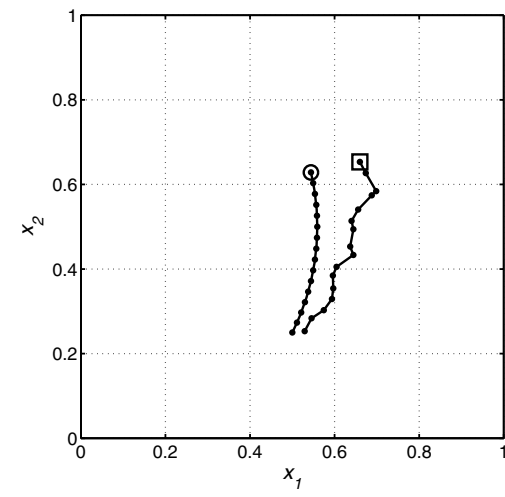

(b)

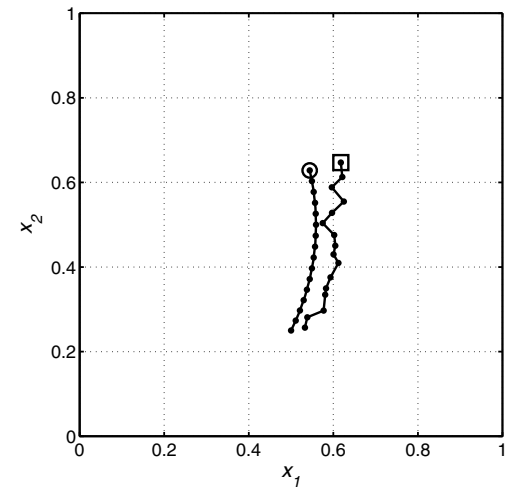

(c)

Fig. 4. Estimated trajectory of the pollution source for two (a), three (b) and four (c) sensors (starting from time $t=150 \mathrm{~s}$ indicated with a square). For comparison, the actual trajectory of the moving source is marked with an open circle.

$\left(t_{k}, t_{k}+600\right]$ as well as the initial conditions estimated from the solution for $\tilde{T}_{k-1}$.

- Once we have the predicted response of our system, the $D_{s}$-optimal controls for the sensor nodes on the interval $\tilde{T}_{k}$ are determined and applied on the interval $T_{k}$.

As regards the sensors dynamics, the model

$$
\dot{s}(t)=u(t), \quad s(0)=s_{0},
$$

was applied, focusing on the direct control of sensor velocities. The following bounds for $u$ were used:

$$
-4.2 \leq u_{i}(t) \leq 4.2[\mathrm{~km} / \mathrm{h}], \quad \forall t \in T
$$

To slightly simplify computations, the constraints regarding collision avoidance were neglected. However, in practice, any sensor network platform possesses some amount of autonomy allowing avoiding such critical situations.
3.1.3. Implementation details. In order to verify the proposed approach, a MATLAB program was written using a PC equipped with a Pentium M740 processor (1.73GHz, 1 GB RAM) running Windows XP and MATLAB 7 (R14). First, the system of PDEs was solved using the UMFPACK solver from the COMSOL 3.4 environment based on the finite-element method. Each time calculations were performed for a spatial mesh composed of 3912 triangles and 2017 nodes and evenly partitioned time interval (100 subintervals) using quadratic Lagrange elements. The sensitivity coefficients were then linearly interpolated and stored. In each consecutive time subinterval the system parameters were estimated using the nlinfit function from the MATLAB Statistics Toolbox. Finally, for determining the optimal trajectories, the package RIOTS_95 was employed. From among its three main optimization procedures, the routine riots was used, which is based on the SQP algorithm.

In order to avoid convergence to local optima, the simulations at each time step were restarted several times 


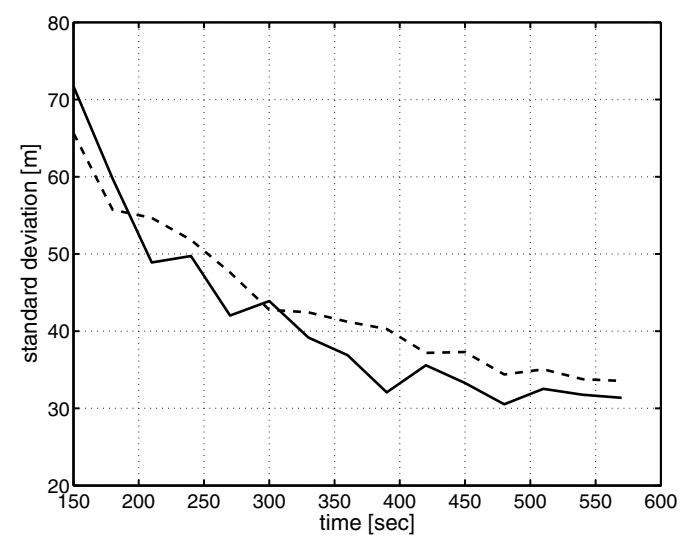

Fig. 5. Estimated standard deviation for the source coordinates in the case of four sensors (solid and dashed lines for $\xi_{k, 1}$ and $\xi_{k, 2}$, respectively).

from different initial starting points. Each simulation run took on average about 60 minutes.

3.1.4. Results. The optimal trajectories are shown in Fig. 3. where the sensor paths are shown for different numbers of nodes. The behavior of sensors is not that intuitive, since the concentration changes are quite complex due to a combination of different mass transport processes. It is clear that sensors attempt to reach the areas with high pollutant concentrations as quickly as possible using their maximum speed in the first part of trajectories. Then the trajectories strongly depend on the number of sensors, since the vehicles tend to cover the area trying to provide the most informative measurements and converge to the source location to terminate in its vicinity. The effects of the presented localization technique are shown in Fig. 4 , where we can easily compare the quality of localization for different numbers of sensors (the last point in the estimated source trajectory is obtained via velocity projection from the starting point from the last subinterval). It becomes clear that an increased number of sensors substantially improves the quality of localization. However, we can also observe the influence of the trajectory modeling error arising from the linearization of the source movements, as the estimated trajectory is located clearly on the righthand side of the real trajectory. This is due to the linear velocity projection leading to an overestimated pollutant concentration on the right-hand side of the domain.

Figure 5 shows the slow improvement in the quality of source localization with the consecutive time steps. This can be explained in terms of better sensor locations at the end of the observation interval (i.e., in a closer vicinity of the moving pollution source) providing more informative measurements.

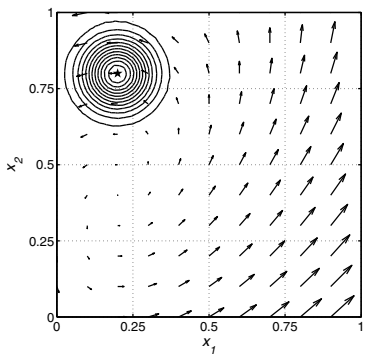

(a) $t=80 \mathrm{~s}$

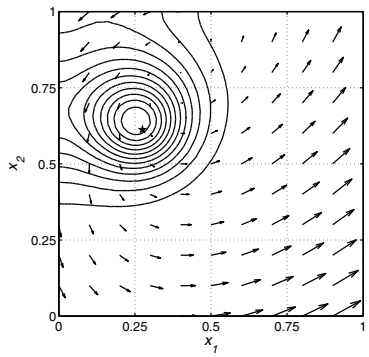

(c) $t=160 \mathrm{~s}$

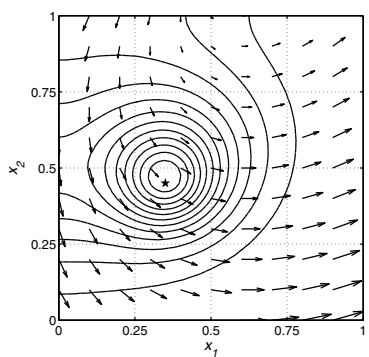

(e) $t=240 \mathrm{~s}$

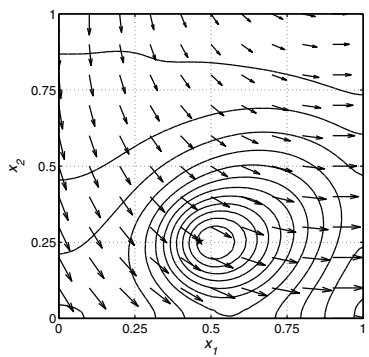

(g) $t=360 \mathrm{~s}$

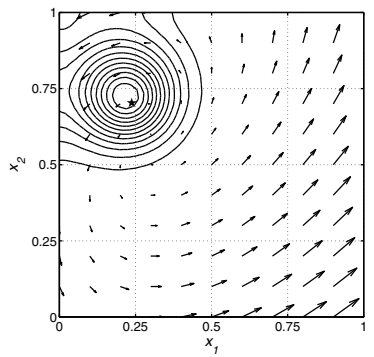

(b) $t=120 \mathrm{~s}$

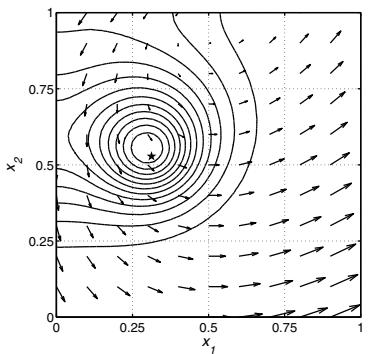

(d) $t=200 \mathrm{~s}$

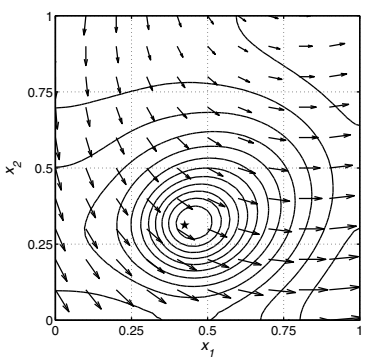

(f) $t=320 \mathrm{~s}$

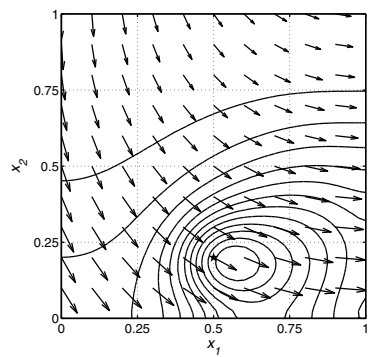

(h) $t=400 \mathrm{~s}$
Fig. 6. Evolution of the wind velocity field versus the pollutant concentration and the actual position of the pollutant source (marked with asterisks).

3.2. Source detection and identification. In this example, the detection and identification of a moving contamination source using information collected with a mobile sensor network will be illustrated via a simulated scenario of pollution expansion over a two-dimensional spatial domain.

3.2.1. Process description. The physical model in our example consists of one source of a pollutant moving inside the spatial domain being a unit square $(1 \mathrm{~km} \times$ 
$1 \mathrm{~km})$. The changes in the spatial concentration $y(x, t)$ of the emitted pollutant over the time interval $T=[0,400]$ (in seconds) are described by the following advectiondiffusion equation:

$$
\begin{aligned}
& \frac{\partial y(x, t)}{\partial t}+\nabla \cdot(v(x, t) y(x, t)) \\
& =\nabla \cdot(\kappa \nabla y(x, t))+f(x, t), \quad(x, t) \in \Omega \times T,
\end{aligned}
$$

subject to the boundary and initial conditions:

$$
\begin{cases}\frac{\partial y(x, t)}{\partial n}=0, & (x, t) \in \Gamma \times T, \\ y(x, 0)=y_{0}, & x \in \Omega\end{cases}
$$

where $\kappa$ denotes a turbulent diffusion coefficient and $\partial y / \partial n$ stands for the partial derivative of $y$ with respect to the outward normal to the boundary $\Gamma$. The term

$$
f(x)=\mathbf{1}\left(t-t_{s}\right) \mu \exp \left(-100\|x-\xi(t)\|^{2}\right),
$$

where $\mathbf{1}(\cdot)$ denotes the unit step function, represents our model of an active source of the pollutant starting emitting the toxic substance at time $t_{s}$ with intensity $\mu$ and the temporal location given by trajectory $\xi(t)$. As for prior estimates of the unknown parameters $\mu$ and $\kappa$, the values 10 $\mathrm{kg} / \mathrm{s}$ and $90 \mathrm{~m}^{2} / \mathrm{s}$ were used, respectively.

3.2.2. Experiment settings. In our simulation scenario, the pollution source is assumed to start the emission at $t_{s}=80 \mathrm{~s}$ from the point $(0.2,0.8)$ and then move along a curve at a time-varying speed to the point $(0.5,0.2)$ attained at $t=400 \mathrm{~s}$. More precisely, its trajectory is given by

$$
\begin{array}{ll}
\xi_{s, 1}=\frac{1}{3200}(3 t+400) & {[\mathrm{km}]} \\
\xi_{s, 2}=5\left(\frac{t-720}{1600}\right)^{2} \quad[\mathrm{~km}]
\end{array}
$$

The wind velocity field $v(x, t)$ is given by the following model (cf. Fig. 6):

$$
v(x, t)=\left(9\left(x_{1}-x_{2}+\frac{t}{400}\right), 9\left(x_{1}-\frac{t}{400}\right)\right) \quad[\mathrm{km} / \mathrm{h}] .
$$

The solution to 64-65) with simulated additive measurement noise with standard deviation $\sigma=10^{-2} \mathrm{~kg} / \mathrm{m}^{3}$ constitutes the reference output signal for our simulations. Our primary purpose here is to detect the presence of the pollution source using a sensor network with $N=4$ mobile nodes. Then, after the detection, we are interested in accurate identification of crucial parameters characterizing the source, e.g., the initial time of the emission, as well as the intensity and current location of the source. Therefore, we have to design the sensor trajectories in such a way as to provide the most informative observations in this context

Due to technical limitations, the measurements are assumed to be taken at multiples of $\Delta t=10 \mathrm{~s}$. Taking this into account, the observation interval is divided into 20 equal design subintervals:

$T_{k}=\left(t_{k}, t_{k}+2 \Delta t\right], \quad t_{k}=2(k-1) \Delta t, \quad k=1, \ldots, 20$.

In such a way, two new observations per sensor are collected in each subinterval.

The detection problem considered here comprises the verification of the null hypothesis $H_{0}: \mu=0$ related to the zero intensity of the source, which is physically equivalent to its absence. To this end, a log-likelihood ratio based technique can be applied (see the work of Patan and Patan (2005) for details). In our simulation scenario, the sensors are assumed to approach the contaminated area starting from the corners of the square domain with half of their maximum velocities in the direction of the center of the area (this time their initial positions are not optimized). Based on the collected measurements, hypothesis $H^{0}$ is verified. Until the source is undetected, the sensors follow paths given a priori.

Under the validity of the alternative hypothesis $H^{1}$ : $\mu>0$ (i.e., the presence of a source), the other source parameters become identifiable. Thus, once the source has been detected, our goal is to properly design the sensor network node movements in order to accurately estimate the source characteristics. In order to slightly simplify parameter estimation, the source trajectory $\xi(t)$ is approximated using linear splines:

$$
\xi(t) \approx \xi_{k}+v_{k}\left(t-t_{k}\right), \quad t \in T_{k}, \quad k=r, \ldots, 20,
$$

where $v_{k}=\left(\xi_{k+1}-\xi_{k}\right) /(2 \Delta t)$ is the average velocity of the source in the interval $T_{k}, \xi_{k}$ denotes the source location at time $t_{k}$ and $t_{r} \geq t_{s}$. Then, for each $T_{k}$, $k=r, \ldots, 20$, we have to update the controls for the sensor network nodes in order to accurately find the current parameters of the source. From among the system parameters $\theta^{k}=\left(\mu, t_{s}, \xi_{k, 1}, \xi_{k, 2}, v_{k, 1}, v_{k, 2}, \kappa\right)$, we are interested in the first four, describing the current source location and intensity. Thus, the maximization of the estimation accuracy for those parameters can be achieved via the determination of $D_{s}$-optimal sensor trajectories.

To reduce the complexity of parameter estimation, a sequential technique was applied to determine the estimates, which can be reflected by the following steps:

- First, the estimate $\hat{\theta}^{k}$ of the current system parameter vector $\theta^{k}$ is determined based on all measurements hitherto collected. The parameters $\mu$ and $t_{s}$ are updated, $\xi_{k}$ is stored as the source position at time $t_{k}, v_{k}$ is used for the prediction of the source location and initial conditions for the system state at the next time instant $t_{k+1}$. 
- The model equations (64) and (65) are solved using (70) and the current estimate $\hat{\theta}^{k}$ on the interval $\tilde{T}_{k}=$ $\left(t_{k}, t_{k}+600\right]$ as well as the initial conditions estimated from the solution for $\tilde{T}_{k-1}$.

- Once we have the predicted response of our system, $D_{s}$-optimal controls for the sensor nodes on the interval $\tilde{T}_{k}$ are determined and applied on the interval $T_{k}$.

As regards the sensors dynamics, the model

$$
\dot{s}(t)=u(t), \quad s(0)=s_{0},
$$

was employed, focusing on the direct control of sensor velocities. The following bounds for $u$ were used:

$$
-11.0 \leq u_{i}(t) \leq 11.0[\mathrm{~km} / \mathrm{h}], \quad \forall t \in T .
$$

3.2.3. Implementation details. In order to verify the proposed approach, a MATLAB program was written using a PC equipped with a Pentium M740 processor (1.73GHz, 1 GB RAM) running Windows XP and MATLAB 7 (R14). First, the system of PDEs was solved using the UMFPACK solver from the COMSOL 3.4 environment exploiting the finite-element method. Each time calculations were performed for a spatial mesh composed of 3912 triangles and 2017 nodes and an evenly partitioned time interval (100 subintervals) using quintic Lagrange elements. The sensitivity coefficients were then linearly interpolated and stored. In each consecutive time subinterval the system parameters were estimated using the nlinfit function from the MATLAB Statistics Toolbox. Finally, for determining the optimal trajectories, the package RIOTS_95 was employed. From among its three main optimization procedures, the routine riots was used, which is based on the SQP algorithm. In order to avoid getting stuck in a local optimum, the simulations were restarted several times from different starting points.

3.2.4. Results. The phase of source detection is presented in Fig. 7, where the test statistic is calculated for each consecutive observation from sensors and compared with a threshold representing a constant false-alarm probability. Because in our scenario we assumed that there is no significant pollutant concentration before the source activation, a likelihood ratio increases in a rather abrupt manner, as soon as one of the sensors measures a reasonably high concentration of the toxic substance. Therefore, for a $5 \%$ false alarm level, we observe almost immediate detection of the source after 20 s, i.e., within two consecutive measurements. Thus, starting from $t=100 \mathrm{~s}$, the identification phase begins when the sensors trajectories are designed sequentially to accurately estimate the source characteristic parameters.

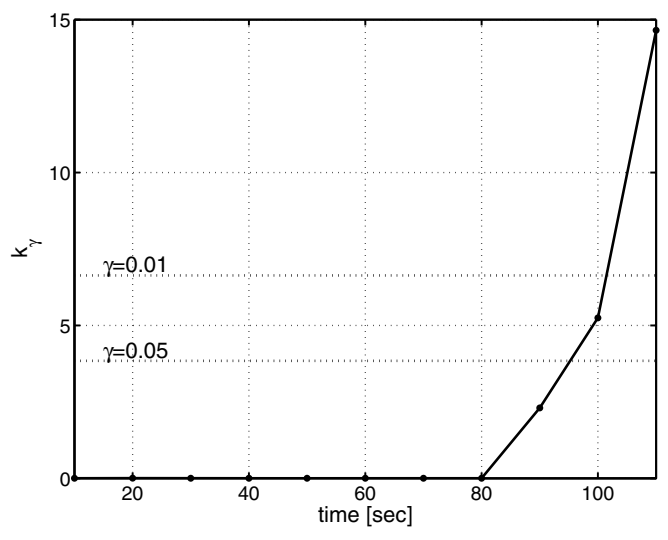

Fig. 7. Log-likelihood test for source detection (indicated thresholds for $5 \%$ and $1 \%$ false alarm levels).

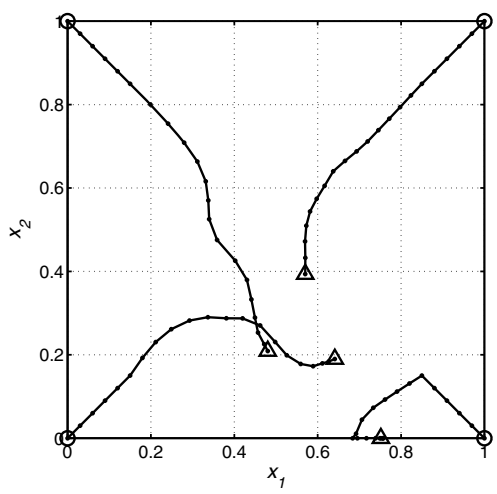

Fig. 8. Optimal sensor trajectories (starting and final positions marked with open circles and triangles, respectively).

The optimal sensor trajectories are shown in Fig. 8 In order to explain the behavior of the sensor network in question we have to compare the sensor paths with Fig. 6 to see that sensors attempt to follow the areas with the greatest temporal concentration changes, which seems to be quite complex due to the combination of different mass transport processes.

The identification results are presented in Figs. 9 and 10. where we can easily compare the estimation quality of the source parameters as well as localization effects.

\section{Conclusions}

In this report we presented results related to the interpretation and use of data in sensor networks with mobile nodes. Specifically, we carried out theoretical research and developed computationally efficient methods and algorithms to determine optimal configurations of mobile sensor networks for contaminating source detection and estimation. What is more, we implemented and tested computer code for the verification of the proposed approach based on simulations. The key assumption made here was that 


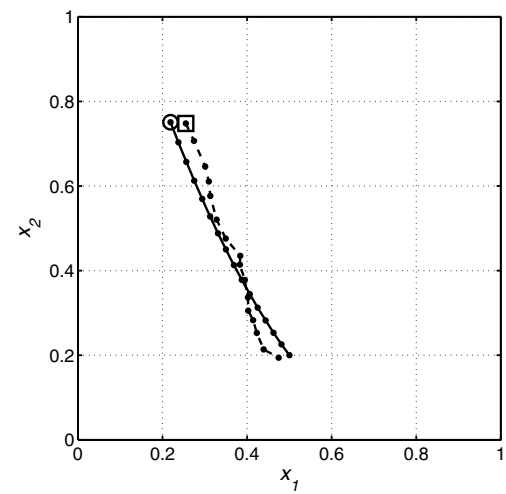

Fig. 9. Estimated trajectory of the pollution source (dashed line) and the actual trajectory (solid line). Their initial positions are indicated with a square and a circle, respectively.

the paths of the moving sources were unknown, but they were sufficiently smooth to be approximated by combinations of given basis functions. The parametrization of the source trajectories made it possible to reduce the source detection and estimation problem to that of parameter identification. In order to estimate the source and medium parameters, the maximum-likelihood estimation was used. Based on a scalar measure of performance defined on the Fisher information matrix related to the unknown parameters, which is commonly used in optimum experimental design, the problem was formulated as an optimal control one with state-variable inequality constraints representing geometric constraints induced by the admissible measurement regions and allowable distances between the sensors. Taking account of the dynamic models of the vehicles carrying the sensors, the problem was reduced to the determination of both the control forces of the sensors and initial sensor positions. This, in turn, was shown to be equivalent to a classical Mayer one, which is thoroughly treated in optimal control theory. The resulting problem involved dynamic constraints in the form of a large-scale system of ordinary differential equations of which some are functionally dependent on the solution of the sensitivity equations associated with the PDE modeling the distributed parameter system in question. Accordingly, numerical solvers of algorithmic optimal control can be employed to solve it. In our approach, we used RIOTS_95, an extremely efficient solver implemented in MATLAB.

The above approach was primarily aimed at off-line design of sensor trajectories. From a practical point of view, it is desirable to have the computations dynamic data driven, i.e., the current measurements from the mobile sensors must serve as a basis for the update of parameter estimates and these, in turn, can be used to correct the sensor movements. This, however, gives rise to a highly nonlinear dynamic optimization problem. Thus, in the proposed research, an attempt was also made at applying a nonlinear receding-horizon approach to attack this issue.

The following is a concise summary of the potential contributions provided by our work to the state-of-the-art in optimal sensor location for source identification in distributed parameter systems:

- The aim of the present work was to develop computationally efficient methods and algorithms to determine optimal trajectories of mobile sensor nodes for source identification in distributed parameter systems. In contrast to conventional approaches commonly used in distributed sensor networks, the knowledge of the mathematical model of the DPS in question is incorporated to form a basis for optimization. Consequently, the important information about the model governing the physical phenomenon in question is not lost and will be to the profit of early detection of potential chemical or biological threats. In numerical examples we used two-dimensional advectiondiffusion partial differential equations, which makes the proposed approach closer to practical applications than most situations considered in the literature.

- Intuitively, we feel that the accuracy of the source identification problem must depend in a way on sensor locations. Numerical experience indicates that this influence is even more dramatic in the case of moving sources and sensors. This fact suggests that we may attempt to select these locations so as to obtain the best estimates of the source positions and intensities. But to form a basis for the comparison of different locations, a quantitative measure of the 'goodness' of particular locations is required. Unfortunately, no measure of this type has been proposed yet. A principal aim of the work was to demonstrate that criteria extensively applied in optimum experimental design and defined on the Fisher information matrix associated with the unknown parameters governing the source motions can be employed for that purpose. Specifically, we employed $\mathrm{D}$ and $\mathrm{D}_{s}$-optimality criteria, which yield minimal volumes of the uncertainty ellipsoids for the estimated parameters. But this does not impose any loss of generality and numerous other criteria can also be used.

- An online version of the proposed method for the design of optimal sensor trajectories could be of paramount importance in the fields of security, environmental and industrial monitoring, pollution control, etc. Motivating examples here include the detection of potential biochemical attacks and detecting leakages of dangerous biochemical materials. To deal with this issue, we have made an attempt at applying a nonlinear approach similar to that used in model predictive control. Simulation results demonstrate that 


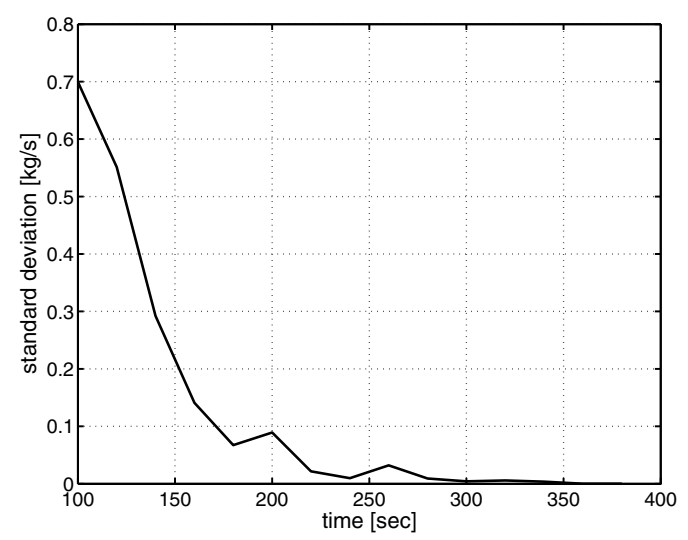

(a)

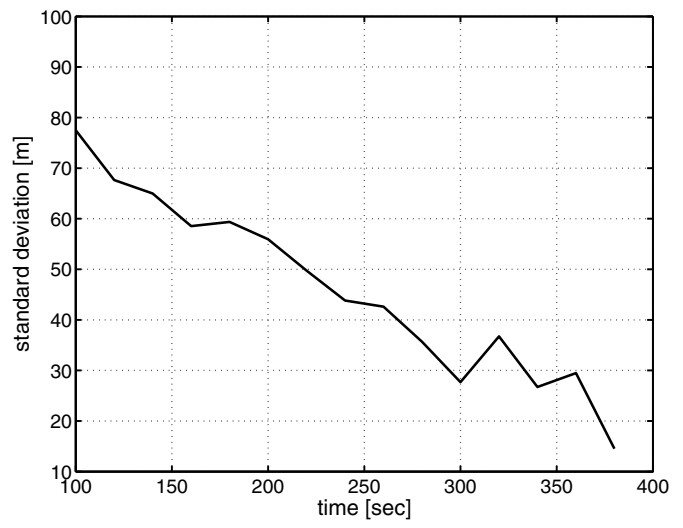

(c)

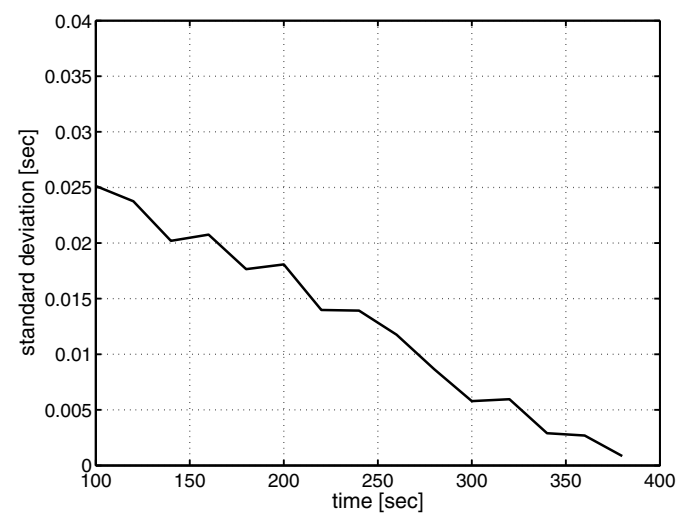

(b)

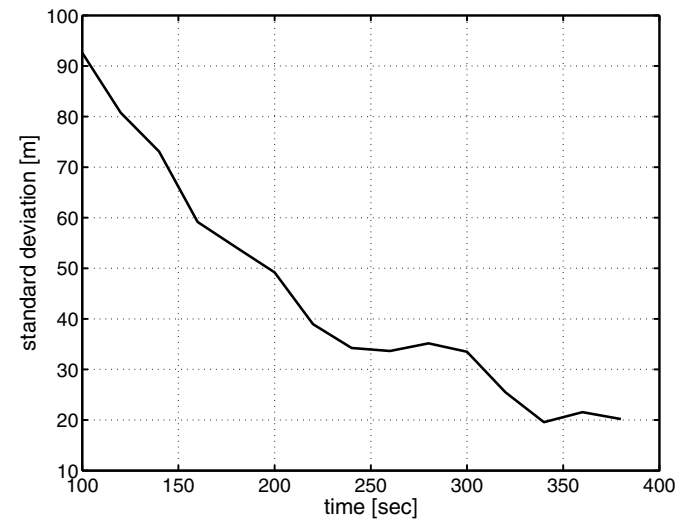

(d)

Fig. 10. Estimated standard deviation for the source parameters: intensity $\mu$ (a), emission starting time $t_{s}$ (b), coordinate $\xi_{1}$ (c), coordinate $\xi_{2}(\mathrm{~d})$.

the resulting scheme behaves well in practice, but a more thorough analysis is required to formally prove its convergence. Unfortunately, this problem is highly nontrivial and seeking its solution is left for future research.

We believe that the ideas presented here are widely applicable to a large number of applications. Basically, the framework can be applied to any problem where there is a spatiotemporal process for which there is an interest in prediction and control and where there is a limited number of sample points available.

\section{Acknowledgment}

This work was supported by the Air Force Office of Scientific Research, Air Force Material Command, USAF, under Grant No. FA8655-08-1-3014, and the Polish Ministry of Science and Higher Education under Grant no. N N519 297133 . The paper is an extended version of the work presented at a plenary session during the 7th Workshop on Advanced Control and Diagnosis, $A C D$ 2009 (Zielona Góra, Poland).

\section{References}

Akçelik, V., Biros, G., Ghattas, O., Long, K. R. and van Bloemen Waanders, B. (2003). A variational finite element method for source inversion for convective-diffusive transport, Finite Elements in Analysis and Design 39: 683-705.

Atkinson, A.C., Donev, A.N. and Tobias, R.D. (2007). Optimum Experimental Designs, with SAS, Oxford University Press, Oxford.

Banks, H.T. (1992). Computational issues in parameter estimation and feedback control problems for partial differential equation systems, Physica D 60: 226-238.

Banks, H.T., Smith, R.C. and Wang, Y. (1996). Smart Material Structures: Modeling, Estimation and Control, Research in Applied Mathematics, Masson, Paris.

Biegler, L.T., Ghattas, O., Heinkenschloss, M., Keyes, D. and van Bloemen Waanders, B. (Eds.) (2007). Real-Time PDEConstrained Optimization, Society for Industrial and Applied Mathematics, Philadelphia, PA.

Boggs, P.T., Long, K.R., Margolis, S.B. and Howard, P.A. (2006). Rapid source inversion for chemical/biological attacks. Part 1: The steady-state case, SIAM Journal on Optimization 17(2): 430-458. 
Butkovskiy, A.G. and Pustyl'nikov, A.M. (1987). Mobile Control of Distributed Parameter Systems, John Wiley \& Sons, New York, NY.

Cassandras, C. G. and Li, W. (2005). Sensor networks and cooperative control, European Journal of Control 11(4-5): 436-463.

Chavent, G. (1991). On the theory and practice of non-linear least-squares, Advances in Water Resources 14(2): 55-63.

Chong, C.-Y. and Kumar, S.P. (2003). Sensor networks: Evolution, opportunities, and challenges, Proceedings of the IEEE 91(8): 1247-1256.

Christofides, P.D. (2001). Nonlinear and Robust Control of PDE Systems: Methods and Applications to Transport-Reaction Processes, Systems \& Control: Foundations \& Applications, Birkhäuser, Boston, MA.

Daescu, D.N. and Navon, I.M. (2004). Adaptive observations in the context of 4D-Var data assimilation, Meteorology and Atmospheric Physics 85: 205-226.

Demetriou, M.A. (2006a). Detection and containment policy of moving source in 2D diffusion processes using sensor/actuator network, Proceedings of the European Control Conference 2007, Kos, Greece, (on CD-ROM).

Demetriou, M.A. (2006b). Power management of sensor networks for detection of a moving source in 2-D spatial domains, Proceedings of the 2006 American Control Conference, Minneapolis, $M N$, (on CD-ROM).

Demetriou, M.A. (2007). Process estimation and moving source detection in 2-D diffusion processes by scheduling of sensor networks, Proceedings of the 2007 American Control Conference, New York, NY, USA, (on CD-ROM).

Demetriou, M.A. (2009). Natural consensus filters for second order infinite dimensional systems, Systems \& Control Letters 58(12): 826-833.

Demetriou, M.A. and Hussein, I.I. (2009). Estimation of spatially distributed processes using mobile spatially distributed sensor network, SIAM Journal on Control and Optimization 48(1): 266-291.

Fedorov, V.V. and Hackl, P. (1997). Model-Oriented Design of Experiments, Lecture Notes in Statistics, Springer-Verlag, New York, NY.

Ford, I., Titterington, D.M. and Kitsos, C.P. (1989). Recent advances in nonlinear experimental design, Technometrics 31(1): 49-60.

Gevers, M. (2005). Identification for control: From the early achievements to the revival of experiment design, European Journal of Control 11(4-5): 335-352.

Gnot, S., Rafajłowicz, E. and Urbańska-Motyka, A. (2001). Statistical inference in a linear model for spatially located sensors and random input, Annals of the Institute of Statistical Mathematics 53(2): 370-379.

Goodwin, G.C. and Payne, R.L. (1977). Dynamic System Identification. Experiment Design and Data Analysis, Mathematics in Science and Engineering, Academic Press, New York, NY.
Gruver, W.A. and Sachs, E. (1980). Algorithmic Methods in Optimal Control, Pitman Publishing Limited, London.

Hirsch, M.J., Pardalos, P.M., Murphey, R. and Grundel, D. (Eds.) (2008). Advances in Cooperative Control and Optimization. Proceedings of the 7th International Conference on Cooperative Control and Optimization, Springer-Verlag, Berlin.

Hjalmarsson, H. (2005). From experiment design to closed-loop control, Automatica 41(3): 393-438.

Hussein, I. I. and Demetriou, M. A. (2007). Estimation of distributed processes using mobile spatially distributed sensors, Proceedings of the 2007 American Control Conference, New York, NY, USA, (on CD-ROM).

Isakov, V. (1998). Inverse Problems for Partial Differential Equations, Applied Mathematical Sciences, Springer-Verlag, New York, NY.

Jacobson, M.Z. (1999). Fundamentals of Atmospheric Modeling, Cambridge University Press, Cambridge.

Jain, N. and Agrawal, D.P. (2005). Current trends in wireless sensor network design, International Journal of Distributed Sensor Networks 1: 101-122.

Jennings, L.S., Fisher, M.E., Teo, K.L. and Goh, C.J. (2002). MISER 3: Optimal Control Software, Version 2.0. Theory and User Manual, Department of Mathematics, University of Western Australia, Nedlands, http: / / www . cado. uwa. edu. au/miser/.

Jeremić, A. and Nehorai, A. (1998). Design of chemical sensor arrays for monitoring disposal sites on the ocean floor, $I E$ EE Transactions on Oceanic Engineering 23(4): 334-343.

Jeremić, A. and Nehorai, A. (2000). Landmine detection and localization using chemical sensor array processing, IEEE Transactions on Signal Processing 48(5): 1295-1305.

Kubrusly, C.S. and Malebranche, H. (1985). Sensors and controllers location in distributed systems-A survey, Automatica 21(2): 117-128.

Kusiak, S. and Weatherwax, J. (2008). Identification and characterization of a mobile source in a general parabolic differential equation with constant coefficients, SIAM Journal on Applied Mathematics 68(3): 784-805.

Lefèvre, F. and Niliot, C.L. (2002). The BEM for point heat source estimation: Application to multiple static sources and moving sources, International Journal of Thermal Sciences 41: 536-546.

Lehmann, E.L. and Romano, J.P. (2005). Testing Statistical Hypotheses, 3rd Edn., Springer-Verlag.

Ljung, L. (1999). System Identification: Theory for the User, 2nd Edn., Prentice Hall, Upper Saddle River, NJ.

Maksimov, V.I. (2000). Problems of Dynamic Input Reconstruction of Infinite-Dimensional Systems, Russian Academy of Sciences Press, Ekaterinburg, (in Russian).

Martínez, S. and Bullo, F. (2006). Optimal sensor placement and motion coordination for target tracking, Automatica 42(4): 661-668. 
Navon, I.M. (1997). Practical and theoretical aspects of adjoint parameter estimation and identifiability in meteorology and oceanography, Dynamics of Atmospheres and Oceans 27: 55-79.

Nehorai, A., Porat, B. and Paldi, E. (1995). Detection and localization of vapor-emitting sources, IEEE Transactions on Signal Processing 43(1): 243-253.

Ögren, P., Fiorelli, E. and Leonard, N.E. (2004). Cooperative control of mobile sensor networks: Adaptive gradient climbing in a distributed environment, IEEE Transactions on Automatic Control 49(8): 1292-1302.

Patan, M. and Patan, K. (2005). Optimal observation strategies for model-based fault detection in distributed systems, International Journal of Control 78(18): 1497-1510.

Patan, M. and Uciński, D. (2005). Optimal activation strategy of discrete scanning sensors for fault detection in distributedparameter systems, Proceedings of the 16th IFAC World Congress, Prague, Czech Republic, (on CD-ROM).

Patan, M. and Uciński, D. (2008). Configuring a sensor network for fault detection in distributed parameter systems, International Journal of Applied Mathematics and Computer Science 18(4): 513-524, DOI: 10.2478/v10006-008-00454.

Polak, E. (1997). Optimization. Algorithms and Consistent Approximations, Applied Mathematical Sciences, SpringerVerlag, New York, NY.

Porat, B. and Nehorai, A. (1996). Localizing vapor-emitting sources by moving sensors, IEEE Transactions on Signal Processing 44(4): 1018-1021.

Pytlak, R. (1999). Numerical Methods for Optimal Control Problems with State Constraints, Springer-Verlag, Berlin.

Quereshi, Z.H., Ng, T.S. and Goodwin, G.C. (1980). Optimum experimental design for identification of distributed parameter systems, International Journal of Control 31(1): 2129.

Rafajłowicz, E. (1981). Design of experiments for eigenvalue identification in distributed-parameter systems, International Journal of Control 34(6): 1079-1094

Rafajłowicz, E. (1983). Optimal experiment design for identification of linear distributed-parameter systems: Frequency domain approach, IEEE Transactions on Automatic Control 28(7): 806-808.

Rafajłowicz, E. (1986). Optimum choice of moving sensor trajectories for distributed parameter system identification, International Journal of Control 43(5): 1441-1451.

Sastry, S. and Iyengar, S.S. (2005). Real-time sensor-actuator networks, International Journal of Distributed Sensor Networks 1: 17-34.

Schwartz, A. L., Polak, E. and Chen, Y. (1997). A Matlab Toolbox for Solving Optimal Control Problems. Version 1.0 for Windows, http: //www. schwartz-home.com / adam/RIOTS/.

Sinopoli, B., Sharp, C., Schenato, L., Schaffert, S. and Sastry, S.S. (2003). Distributed control applications within sensor networks, Proceedings of the IEEE 91(8): 1235-1246.
Sivergina, I.F. and Polis, M.P. (2002). Comments on "Modelbased solution techniques for the source localization problem", IEEE Transactions on Control Systems Technology 10(4): 633-633.

Sivergina, I.F., Polis, M.P. and Kolmanovsky, I. (2003). Source identification for parabolic equations, Mathematics of Control, Signals, and Systems 16: 141-157.

Song, Z., Chen, Y., Sastry, C. R. and Tas, N. C. (2009). Optimal Observation for Cyber-physical Systems: A FisherInformation-Matrix-Based Approach, Springer-Verlag, London.

Sun, N.-Z. (1994). Inverse Problems in Groundwater Modeling, Theory and Applications of Transport in Porous Media, Kluwer Academic Publishers, Dordrecht.

Uciński, D. (1999). Measurement Optimization for Parameter Estimation in Distributed Systems, Technical University Press, Zielona Góra.

Uciński, D. (2000a). Optimal selection of measurement locations for parameter estimation in distributed processes, International Journal of Applied Mathematics and Computer Science 10(2): 357-379.

Uciński, D. (2000b). Optimal sensor location for parameter estimation of distributed processes, International Journal of Control 73(13): 1235-1248.

Uciński, D. (2005). Optimal Measurement Methods for Distributed-Parameter System Identification, CRC Press, Boca Raton, FL.

Uciński, D. and Atkinson, A. C. (2004). Experimental design for time-dependent models with correlated observations, Studies in Nonlinear Dynamics \& Econometrics 8(2), Article No. 13.

Uciński, D. and Bogacka, B. (2005). T-optimum designs for discrimination between two multivariate dynamic models, Journal of the Royal Statistical Society: Series B (Statistical Methodology) 67: 3-18.

Uciński, D. and Chen, Y. (2005). Time-optimal path planning of moving sensors for parameter estimation of distributed systems, Proceedings of the 44th IEEE Conference on Decision and Control, and the European Control Conference 2005, Seville, Spain, (on CD-ROM).

Uciński, D. and Chen, Y. (2006). Sensor motion planning in distributed parameter systems using Turing's measure of conditioning, Proceedings of the 45th IEEE Conference on Decision and Control, San Diego, CA, USA, (on CD$\mathrm{ROM})$.

Uciński, D. and Demetriou, M.A. (2008). Resource-constrained sensor routing for optimal observation of distributed parameter systems, Proceedings of the 18th International Symposium on Mathematical Theory of Networks and Systems, Blacksburg, VA, (on CD-ROM).

Uciński, D. and Korbicz, J. (2001). Optimal sensor allocation for parameter estimation in distributed systems, Journal of Inverse and Ill-Posed Problems 9(3): 301-317.

Uciński, D. and Patan, M. (2007). D-optimal design of a monitoring network for parameter estimation of distributed systems, Journal of Global Optimization 39: 291-322. 
Uspenskii, A.B. and Fedorov, V.V. (1975). Computational Aspects of the Least-Squares Method in the Analysis and Design of Regression Experiments, Moscow University Press, Moscow, (in Russian).

van de Wal, M. and de Jager, B. (2001). A review of methods for input/output selection, Automatica 37(4): 487-510.

Vogel, C.R. (2002). Computational Methods for Inverse Problems, Frontiers in Applied Mathematics, Society for Industrial and Applied Mathematics, Philadelphia, PA.

von Stryk, O. (1999). User's Guide for DIRCOL, a Direct Collocation Method for the Numerical Solution of Optimal Control Problems. Version 2.1, Simulation, Systems Optimization and Robotics Group, Technical University of Darmstadt.

http://www.sim.informatik.

//tu-darmstadt.de/index/leftnav.html.en.

Walter, É. and Pronzato, L. (1990). Qualitative and quantitative experiment design for phenomenological models-A survey, Automatica 26(2): 195-213.

Walter, É. and Pronzato, L. (1997). Identification of Parametric Models from Experimental Data, Communications and Control Engineering, Springer-Verlag, Berlin.

Zhao, F. and Guibas, L.J. (2004). Wireless Sensor Networks: An Information Processing Approach, Morgan Kaufmann Publishers, Amsterdam.

Zhao, T. and Nehorai, A. (2006). Detecting and estimating biochemical dispersion of a moving source in a semiinfinite medium, IEEE Transactions on Signal Processing 54(6): 2213-2225.

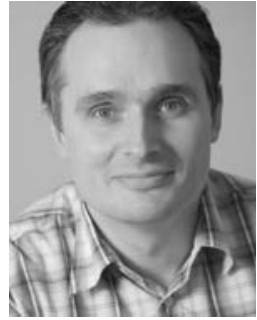

Dariusz Uciński was born in 1965 . He received the M.Sc. degree in electrical engineering from the Higher College of Engineering in Zielona Góra, Poland, in 1989, and the Ph.D. and D.Sc. degrees in automatic control and robotics from the Technical University of Wrocław, Poland, in 1992 and 2000, respectively. In 2007 he was conferred the professorial title, the highest scientific degree in Poland. He is currently a professor at the University of Zielona Góra, Poland. His research interests are in the area of measurement optimization for distributed parameter systems. He authored the book entitled Optimal Measurement Methods for Distributed Parameter System Identification (CRC Press, 2005). Other areas of his expertise include optimum experimental design, algorithmic optimal control, robotics and parallel computing. Since 1992 he has served as the deputy editor of the International Journal of Applied Mathematics and Computer Science. Since 2008 he has been the chair of the Control Systems Society Chapter of the IEEE Poland Section.

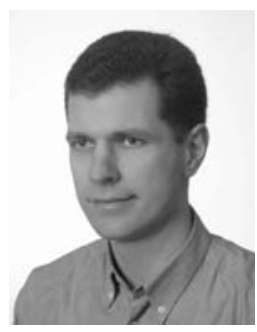

Maciej Patan was born in 1975. He received his M.Sc. and Ph.D degrees, both in electrical engineering, from the University of Zielona Góra, Poland, in 2000 and 2004, respectively. Since 2004 he has been an assistant professor with the Faculty of Electrical Engineering, Computer Science and Telecommunications, University of Zielona Góra. From September 2005 to March 2006, he was a research assistant in statistics at the School of Mathematical Sciences, Queen Mary, University of London. He has been an IEEE member since 2009. His research interests include optimal experimental design in distributed parameter systems, distributed computing and optimization in sensor networks, system identification, and mobile robotics.

Received: 11 February 2010 Revised: 30 March 2010 